\title{
Anomaly mediated SUSY breaking scenarios in the light of cosmology and in the dark (matter)
}

\author{
Alexandre Arbey, ${ }^{a, b, c, d}$ Aldo Deandrea ${ }^{a}$ and Ahmad Tarhini ${ }^{a}$ \\ ${ }^{a}$ Université de Lyon, Université Lyon 1, CNRS/IN2P3, UMR5822 IPNL, \\ F-69622 Villeurbanne Cedex, France \\ ${ }^{b}$ CERN Theory Division, Physics Department, \\ CH-1211 Geneva 23, Switzerland \\ ${ }^{c}$ Centre de Recherche Astrophysique de Lyon, Observatoire de Lyon, CNRS, UMR 5574, \\ 9 avenue Charles André, Saint-Genis Laval cedex, F-69561, France \\ ${ }^{d}$ Laboratoire de Physique, Ecole Normale Supérieure de Lyon, \\ 46 allée d'Italie 69007 Lyon, France \\ E-mail: alexandre.arbey@ens-lyon.fr, deandrea@ipnl.in2p3.fr, \\ tarhini@ipnl.in2p3.fr
}

ABSTRACT: Anomaly mediation is a popular and well motivated supersymmetry breaking scenario. Different possible detailed realisations of this set-up are studied and actively searched for at colliders. Apart from limits coming from flavour, low energy physics and direct collider searches, these models are usually constrained by the requirement of reproducing the observations on dark matter density in the universe. We reanalyse these bounds and in particular we focus on the dark matter bounds both considering the standard cosmological model and alternative cosmological scenarios. These scenarios do not change the observable cosmology but relic dark matter density bounds strongly depend on them. We consider few benchmark points excluded by standard cosmology dark matter bounds and suggest that loosening the dark matter constraints is necessary in order to avoid a too strong (cosmological) model dependence in the limits that are obtained for these models. We also discuss briefly the implications for phenomenology and in particular at the Large Hadron Collider.

KEYWORDS: Supersymmetry Phenomenology 


\section{Contents}

1 Introduction 1

2 Anomaly mediated symmetry breaking in the MSSM 2

2.1 Minimal AMSB 3

2.2 HCAMSB 4

$\begin{array}{lll}2.3 & \text { MMAMSB } & 7\end{array}$

3 Anomaly mediated symmetry breaking in the NMSSM $\quad 7$

$\begin{array}{llr}3.1 \text { mNAMSB } & 12\end{array}$

$\begin{array}{llr}3.2 & \text { NHCAMSB } & 12\end{array}$

$\begin{array}{lll}3.3 & \text { NMMAMSB } & 12\end{array}$

4 AMSB and relic density in alternative cosmology 12

$\begin{array}{lll}4.1 & \text { Alternative cosmological scenarios } & 13\end{array}$

$\begin{array}{lll}4.2 & \text { Quintessence } & 18\end{array}$

$\begin{array}{ll}4.3 \text { Late decaying inflaton } & 19\end{array}$

$\begin{array}{ll}4.4 & \text { Primordial entropy production } \\ & 20\end{array}$

$\begin{array}{ll}4.5 & \text { Late reheating }\end{array}$

4.6 BBN constraints and modified relic density 21

$5 \quad$ Generalised relic density constraints $\quad 23$

$6 \quad$ LHC phenomenology $\quad 23$

$\begin{array}{lll}6.1 & \text { mAMSB point A } & 26\end{array}$

$\begin{array}{lll}6.2 & \text { mAMSB point B } & 29\end{array}$

6.3 HCAMSB point C 30

6.4 MMAMSB point D 30

6.5 MMAMSB point E 31

6.6 mNAMSB point $\mathrm{F} \quad 31$

$\begin{array}{lll}7 & \text { Conclusions and perspectives } & 31\end{array}$

\section{Introduction}

The search for supersymmetry and its breaking, in addition to the direct searches at LEP, B-factories, Tevatron and the Large Hadron Collider (LHC), is actively pursued using the WMAP limits on the relic density constraints. However the sensitivity of the lightest supersymmetric particle relic density calculation to the variation of the cosmological expansion rate before Big-Bang Nucleosynthesis (BBN), even if modest and with no consequences on the cosmological observations, can modify considerably the relic density, and therefore 
change the constraints on the supersymmetric parameter space [1-3]. In the standard cosmology the dominant component before $\mathrm{BBN}$ is radiation, however energy density and entropy content can be modified. In the following we consider the impact of different scenarios of alternative cosmologies. The precision of the WMAP data should therefore not make us forget the hypothesis which are implied by the use of standard cosmology. We discuss in the following the implications of precision B-physics, direct searches and cold dark matter relic abundance for the case of anomaly mediated models, from a minimal anomaly mediation supersymmetry breaking [4-8], to mixed moduli-anomaly mediated [9] and to hypercharge anomaly mediation [10]. We also discuss similar supersymmetry breaking scenarios in the case of the next-to-minimal supersymmetric standard model. For a detailed discussion of anomaly mediation and cosmology see [11]. In section 2 we discuss the different anomaly mediated supersymmetry breakings in the Minimal Supersymmetric Standard Model (MSSM) in terms of the parameter spaces for these models and discuss the limits which can be applied due to the present data from particle physics experiments and from relic dark matter density assuming the standard model of cosmology. In section 3 similar scenarios are considered in the Next-to-Minimal Supersymmetric Standard Model (NMSSM) and the corresponding particle and cosmological bounds are discussed. In section 4 we discuss how alternative cosmological models can affect dramatically the bounds on the parameter space of the models we have considered while letting unchanged the observable cosmology. Four different alternatives to the standard cosmology are discussed which share this behaviour. Section 5 and section 6 discuss respectively the constraints implied by these different cosmological scenarios and the perspectives at the LHC for a list of benchmark points which are representative of the available parameter space for these AMSB models. Our conclusions are given in section 7 .

\section{Anomaly mediated symmetry breaking in the MSSM}

The superconformal Anomaly Mediated Supersymmetry Breaking (AMSB) mechanism [48] is one of the most well-known and attractive set-ups for supersymmetry breaking. Supersymmetry breaking effects in the observable sector have in this framework a gravitational origin. Superconformal symmetry is classically preserved in theories without dimensional parameters and it is in general broken by the quantum effects. As anomalies only depend on the low-energy effective theory, the same will be true for the soft terms. Usually the AMSB scenario cannot be applied to the MSSM, as it leads to tachyonic sleptons. However the presence of an intermediate threshold can displace the soft terms and avoid this problem. In superconformal gravity one introduces a chiral superfield playing the role of the compensating multiplet for super-Weyl transformations, called the Weyl or conformal compensator. The F-term vacuum expectation value of the conformal compensator is turned on by the supersymmetry breaking in the hidden sector and the soft breaking of supersymmetry in the visible sector appears through the chiral anomaly supermultiplet. As the soft SUSY breaking terms arise from the anomaly, the supersymmetry breaking terms do not dominate at tree-level. Several soft SUSY breaking scenarios can be realised starting from this setup. We discuss in the following some of these realisations. 


\subsection{Minimal AMSB}

The minimal AMSB (mAMSB) scenario [4-8] has very attractive properties, since the soft SUSY breaking terms are calculated in terms of one single parameter, namely the gravitino mass $m_{3 / 2}$, and the soft terms are renormalisation group invariants which can be calculated for any scale choice. However, the AMSB scenarios suffer from the problem that slepton squared masses are found to be negative, leading to tachyonic states. A solution to this problem is to consider that the scalar particles acquire a universal mass $m_{0}$ at the GUT scale, which when added to the AMSB soft SUSY breaking terms, makes them positive. Therefore, the mAMSB model relies on only four parameters:

$$
m_{0}, m_{3 / 2}, \tan \beta, \operatorname{sgn}(\mu) .
$$

This scenario has been thoroughly studied in the literature, but is known to have cosmological consequences incompatible with the WMAP observations of the dark matter density [12]. We perform here an updated analysis of the mAMSB parameter space constraints from flavour physics and cosmological relic density. For this study, we generate mass spectra and couplings using Isajet 7.80 [13]. The calculation of flavour observables and the computation of the relic density are performed with SuperIso Relic v3.0 [14-18]. We use the constraints given in table 9 of the latest version of the SuperIso manual.

The first flavour observable that we consider here is the branching ratio of $B \rightarrow X_{s} \gamma$, which has been been thoroughly studied in the literature and is still under scrutiny. This observable is very interesting, as its SM contributions only appear at loop level, and its theoretical uncertainties as well as the experimental errors are now under control. It provides strong constraints on the supersymmetric parameter space, especially for large $\tan \beta$, where it receives large enhancements from its supersymmetric contributions. We use the following interval at 95\% C.L. $[14-16,19]$ :

$$
2.16 \times 10^{-4}<\operatorname{BR}\left(B \rightarrow X_{s} \gamma\right)<4.93 \times 10^{-4}
$$

Another interesting observable is the branching fraction of $B_{s} \rightarrow \mu^{+} \mu^{-}$, which is also a loop level observable, and which can receive extremely large contributions from SUSY at large $\tan \beta$, and can receive an enhancement of several orders of magnitude compared to the SM branching ratio. This decay mode has not yet been observed, and we have at $95 \%$ C.L. $[14-16,20]$ :

$$
\mathrm{BR}\left(B_{s} \rightarrow \mu^{+} \mu^{-}\right)<4.7 \times 10^{-8} .
$$

We also consider a set of tree-level observables which are very sensitive to the charged Higgs mass as well as $\tan \beta$, and we use the following $95 \%$ level intervals, which include 
the theoretical and experimental errors [14-16, 21, 22]:

$$
\begin{aligned}
0.56 & <\frac{\mathrm{BR}(B \rightarrow \tau \nu)}{\mathrm{BR}_{S M}(B \rightarrow \tau \nu)}<2.70, \\
4.7 \times 10^{-2} & <\mathrm{BR}\left(D_{s} \rightarrow \tau \nu\right)<6.1 \times 10^{-2}, \\
0.151 & <\frac{\mathrm{BR}\left(B \rightarrow D^{0} \tau \nu\right)}{\operatorname{BR}\left(B \rightarrow D^{0} e \nu\right)}<0.681, \\
0.982 & <\operatorname{R}_{\ell 23}(K \rightarrow \mu \nu)<1.018 .
\end{aligned}
$$

The observable $\mathrm{R}_{\ell 23}(K \rightarrow \mu \nu)$ is related to the decay of $K \rightarrow \mu \nu$ and is detailed in [22]. For the relic density constraint, we use the WMAP constraints [23] increased by $10 \%$ of theoretical error to account for the uncertainties in the calculation of the relic density:

$$
0.088<\Omega_{D M} h^{2}<0.123
$$

In the following, we disregard the case of negative $\operatorname{sgn}(\mu)$ since it is disfavoured by the muon anomalous magnetic moment constraint, and we scan over the intervals $m_{0} \in[0,2000] \mathrm{GeV}$, $m_{3 / 2} \in[0,100] \mathrm{TeV}$ and $\tan \beta \in[0,60]$. Figure 1 presents projection plots of the parameter space into the possible different planes. The green region in the plots corresponds to the parameter zone which is not excluded by flavour constraints or mass limits. The red stars corresponds to points leading to a favoured relic density but excluded by other constraints, whereas black stars are favoured by all the presented constraints, including the relic density constraint. As can be seen, no black star is visible in these plots, and the whole parameter space presented here is disfavoured either by flavour or direct constraints, or by the relic density constraint which tends to favour the low $m_{3 / 2}$ region. Disregarding the relic density constraint, a large zone at low $m_{3 / 2}$ is excluded.

In figure 2, we show the relic density values as a function of the AMSB parameters. The green zones correspond to regions favoured by the flavour and direct constraints, whereas the other points are either excluded by these constraints or by cosmological considerations (charged relic or sneutrino relic, which interact therefore strongly). Two green zones clearly appear on the $m_{0}$ and $\tan \beta$ plots, for $\Omega h^{2}$ around $10^{-4}$ and $10^{-9}$. These areas are far from the WMAP dark matter allowed interval, making the mAMSB scenario disfavoured by the standard cosmology.

\subsection{HCAMSB}

Another possibility to solve the negative slepton squared masses of the original AMSB scenario has been proposed: the hypercharge anomaly mediated supersymmetry breaking (HCAMSB) scenario [10], in which the MSSM resides on a D-brane and the hypercharge gaugino mass is generated in a geometrically separated hidden sector [24]. In this way, additional contribution to the gaugino mass $M_{1}$ is generated, and the large value of $M_{1}$ then increases the weak scale slepton masses beyond tachyonic values, solving the generic AMSB problem [10]. 

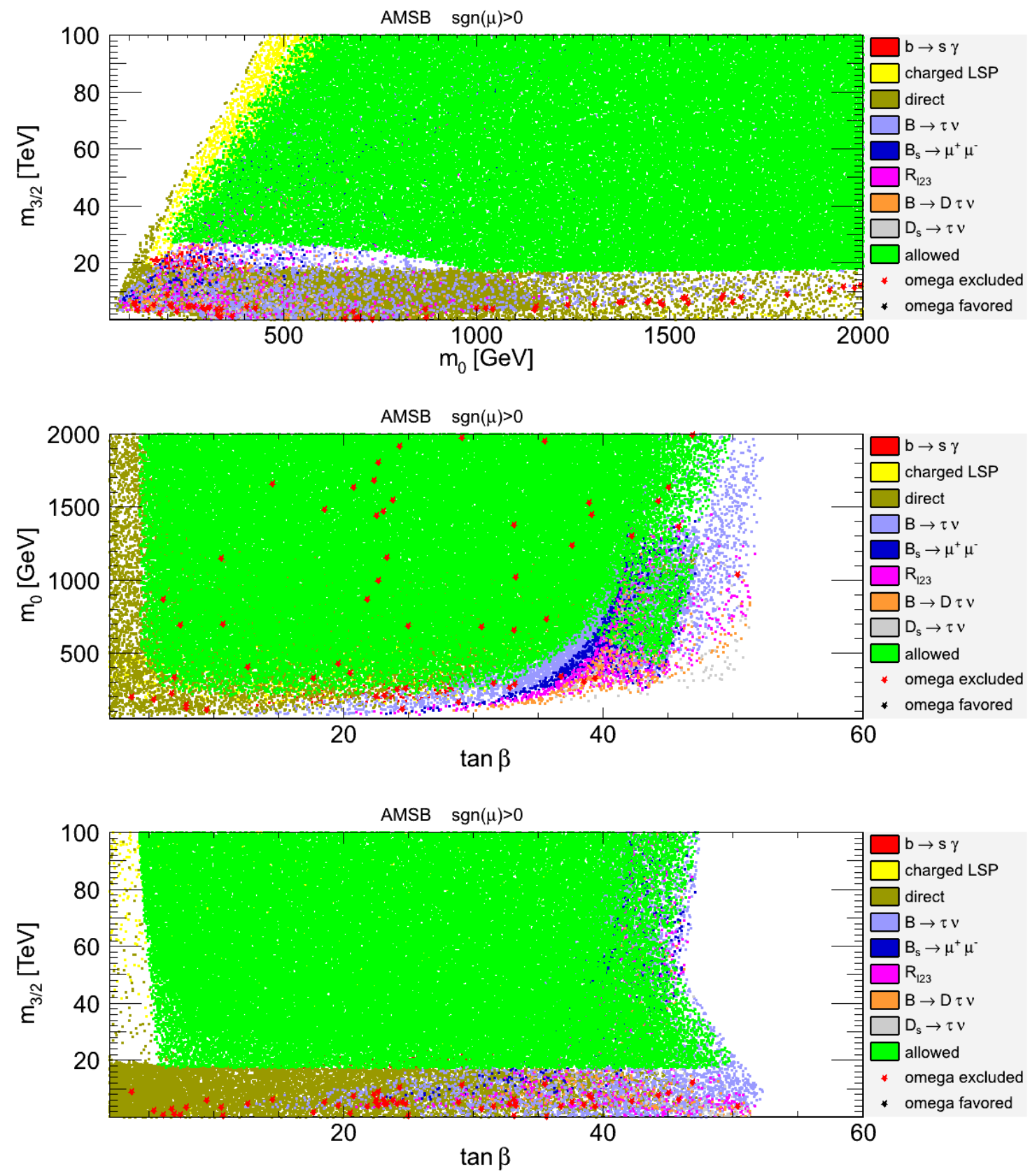

Figure 1. Constraints on the minimal AMSB parameter space. The exclusion regions are plotted in the order given in the legend. The red zones are excluded by the inclusive branching ratio of $B \rightarrow X_{s} \gamma$, the yellow ones correspond to charged LSP, the olive-green areas are excluded by direct collider constraints, the light blue zones are excluded by $\mathrm{BR}(B \rightarrow \tau \nu)$, the dark blue zones by $\operatorname{BR}\left(B_{s} \rightarrow \mu^{+} \mu^{-}\right)$, the magenta zones by $R_{\ell 23}$, the orange zones by $\operatorname{BR}(B \rightarrow D \tau \nu)$ and the grey zones by $\operatorname{BR}\left(D_{s} \rightarrow \tau \nu\right)$. The green area are in agreement with all the previously mentioned constraints. The stars are points favoured by the relic density observable, in red if disfavoured by any other constraints and in black if in agreement with all the constraints simultaneously. 

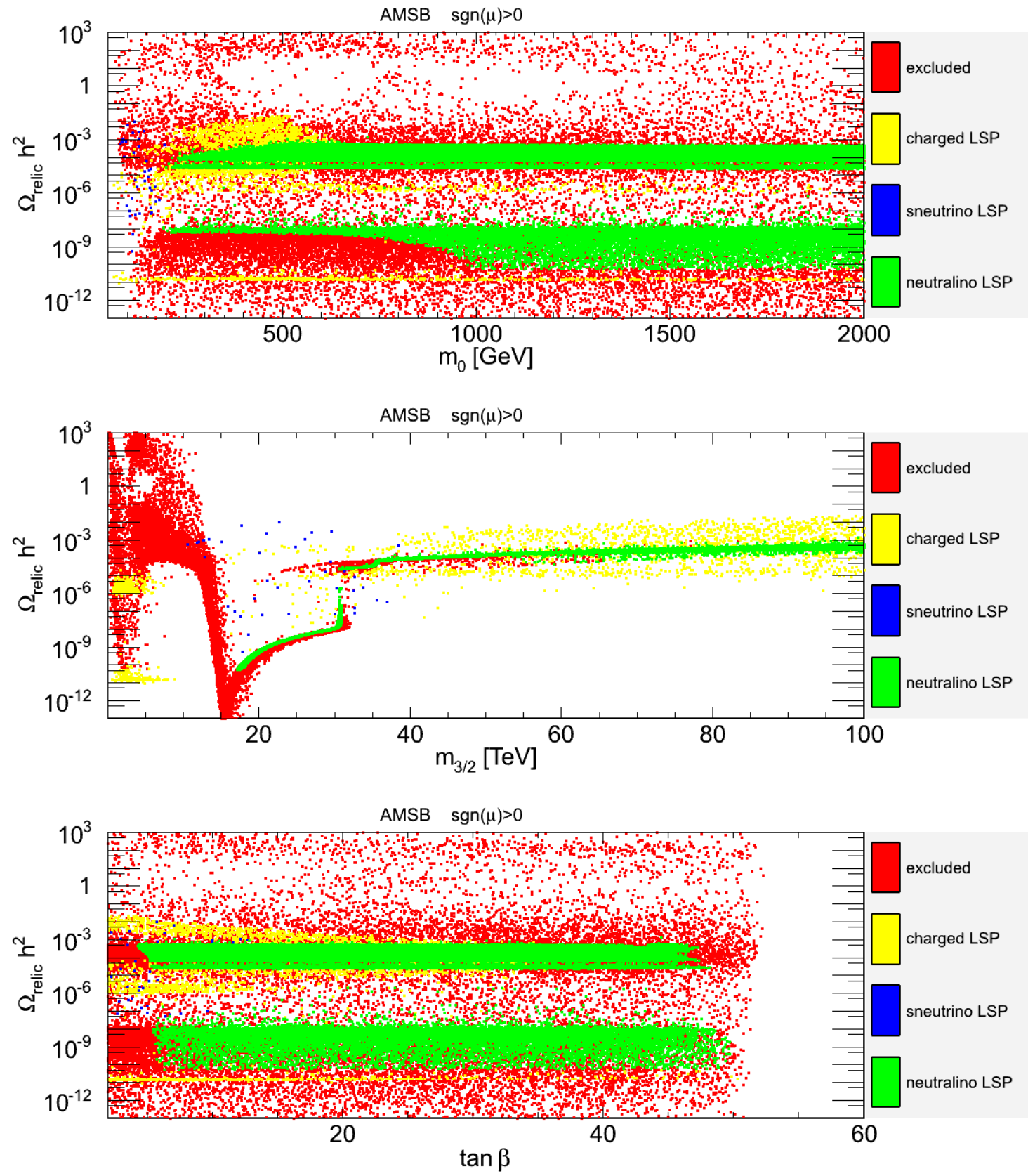

Figure 2. Relic density in function of the AMSB parameters. The green points are favoured by all the constraints, the yellow points corresponds to a charged LSP, the blue points correspond to left sneutrino LSP, and the red points are excluded by the other constraints (flavour and direct limits).

The HCAMSB scenario has four parameters:

$$
\alpha=\frac{\tilde{M}_{1}}{m_{3 / 2}}, m_{3 / 2}, \tan \beta, \operatorname{sgn}(\mu) .
$$

where $\tilde{M}_{1}$ is the HCAMSB contribution to $M_{1}$. 
In order to study the parameter space, we generate mass spectra and couplings using Isajet 7.80 [13] and compute the flavour observables and relic density with SuperIso Relic v3.0 [14-18]. We use the constraints described in the previous subsection.

In figure 3, we scan over the whole parameter space, and project the results in twodimensional planes. The results are somehow similar to those of the mAMSB scenario: the constraints exclude low $m_{3 / 2}$ values. A large part of the parameter space is favoured by flavour and direct constraints, but unfortunately no part of the parameter space respects at the same time the relic density and the other constraints, so that HCAMSB is also disfavoured by the standard cosmology.

In figure 4, we show the relic density values as a function of the HCAMSB parameters. Again, the results are similar to those of the mAMSB scenario, with two distinct zones which are not excluded by flavour and direct constraints, corresponding to a relic density $\Omega h^{2}$ around $10^{-4}$ and $10^{-8}$.

\subsection{MMAMSB}

Contrary to the two previous AMSB scenarios, the Mixed Modulus Anomaly mediated SUSY breaking (MMAMSB) scenario [9] provides viable dark matter candidates, in addition to solving the negative slepton mass problem naturally. This scenario is based on typeIIB superstrings with stabilised moduli [25]. In this scenario, an interesting result is that the soft SUSY breaking terms receive comparable contributions from both anomaly and modulus, resulting in positive slepton masses. We examine here the minimal MMAMSB scenario, ${ }^{1}$ which relies on four parameters:

$$
\alpha, m_{3 / 2}, \tan \beta, \operatorname{sgn}(\mu) .
$$

$\alpha$ here parametrises the relative contributions of modulus mediation and anomaly mediation to the soft breaking terms: the largest $\alpha$ is, the more mediation comes from modulus [9].

In figure 5, the parameter space is scanned over, and it is projected in two-dimensional planes. The resulting plots are different from those of mAMSB and HCAMSB. Indeed, a large part of parameter space escapes the flavour and direct constraints, and zones around $\alpha \sim 0$ or at low $m_{3 / 2}$ also fulfil the relic density constraint.

In figure 6, we present the relic density values in function of the MMAMSB parameters. The relic density of points favoured by flavour and direct constraints takes values between $10^{-9}$ and $10^{3}$. We can notice however that most of the points are in the interval $\left[1,10^{3}\right]$, and some points fit in the WMAP interval. For this reason, MMAMSB is a scenario which can appear as attractive, as it fulfils simultaneously the standard cosmology and particle physics constraints.

\section{Anomaly mediated symmetry breaking in the NMSSM}

An interesting extension of the MSSM is the NMSSM, which brings a solution to the $\mu$-problem [26, 27]. It has an extended Higgs sector involving additional Higgs bosons,

\footnotetext{
${ }^{1}$ We note that in the general MMAMSB, there are two more parameters, $n_{i}$ and $l_{\alpha}$, which represent respectively the modular weights of visible sector of the matter fields and the gauge kinetic function, and which can modify the mass spectra.
} 

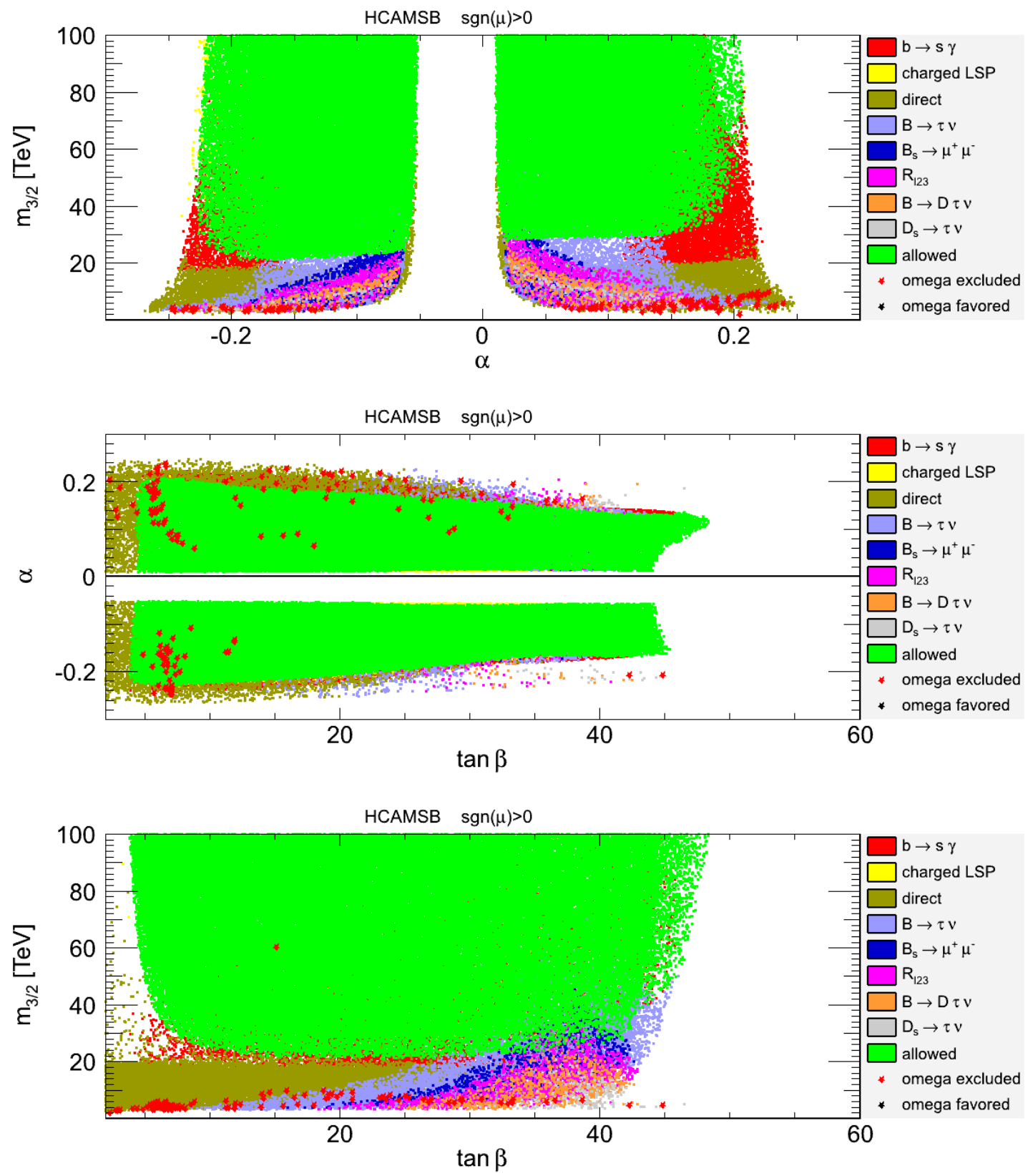

Figure 3. Constraints on the HCAMSB parameter space. The colour codes are the same as in figure 1.

modifying the relic density and flavour physics constraints. Moreover, the couplings being modified, the NMSSM can escape direct constraints, and new parameter zones can be allowed by the constraints used in the previous section.

We consider here the simplest version of the NMSSM, where the term $\mu \hat{H}_{u} \cdot \hat{H}_{d}$ of the MSSM superpotential is replaced by

$$
\lambda \hat{H}_{u} \cdot \hat{H}_{d} \hat{S}+\frac{\kappa}{3} \hat{S}^{3}
$$



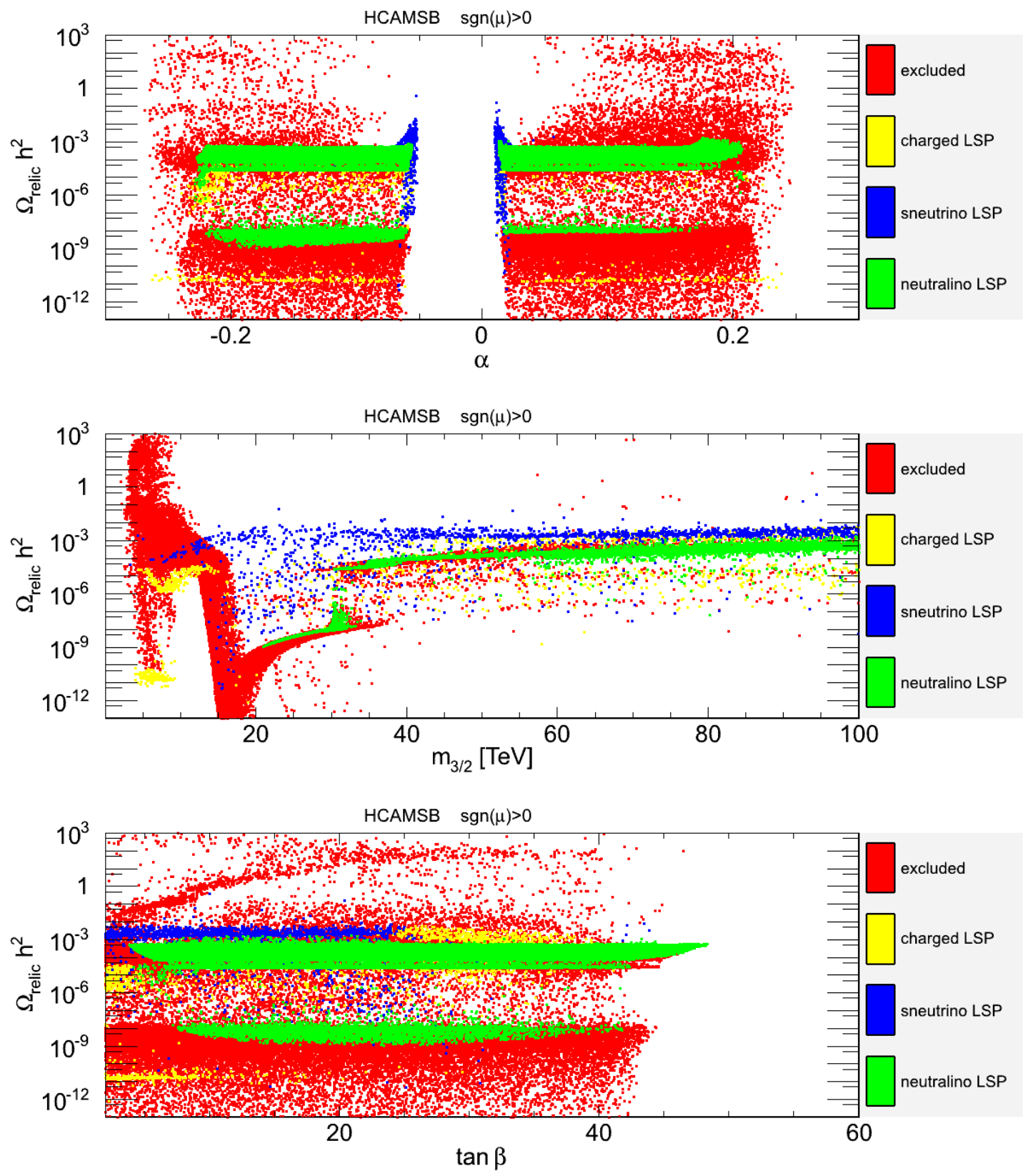

Figure 4. Relic density in function of the HCAMSB parameters. The colour codes are the same as in figure 2 .

in order for the superpotential to be scale invariant. The soft breaking terms

$$
m_{H_{u}}^{2}\left|H_{u}\right|^{2}+m_{H_{d}}^{2}\left|H_{d}\right|^{2}+m_{S}^{2}|S|^{2}+\left(\lambda A_{\lambda} S H_{u} \cdot H_{d}+\frac{1}{3} \kappa A_{\kappa} S^{3}+\text { h.c. }\right),
$$

are a priori independent. Using the minimisation conditions for the potential, the scalar mass parameters $m_{H_{u, d}}$ can be replaced by the vacuum expectation values of the doublet $v_{u}$ and $v_{d}$, with

$$
v_{u}^{2}+v_{d}^{2}=v^{2} \approx(174 \mathrm{GeV})^{2}, \quad \tan \beta=\frac{v_{u}}{v_{d}} .
$$



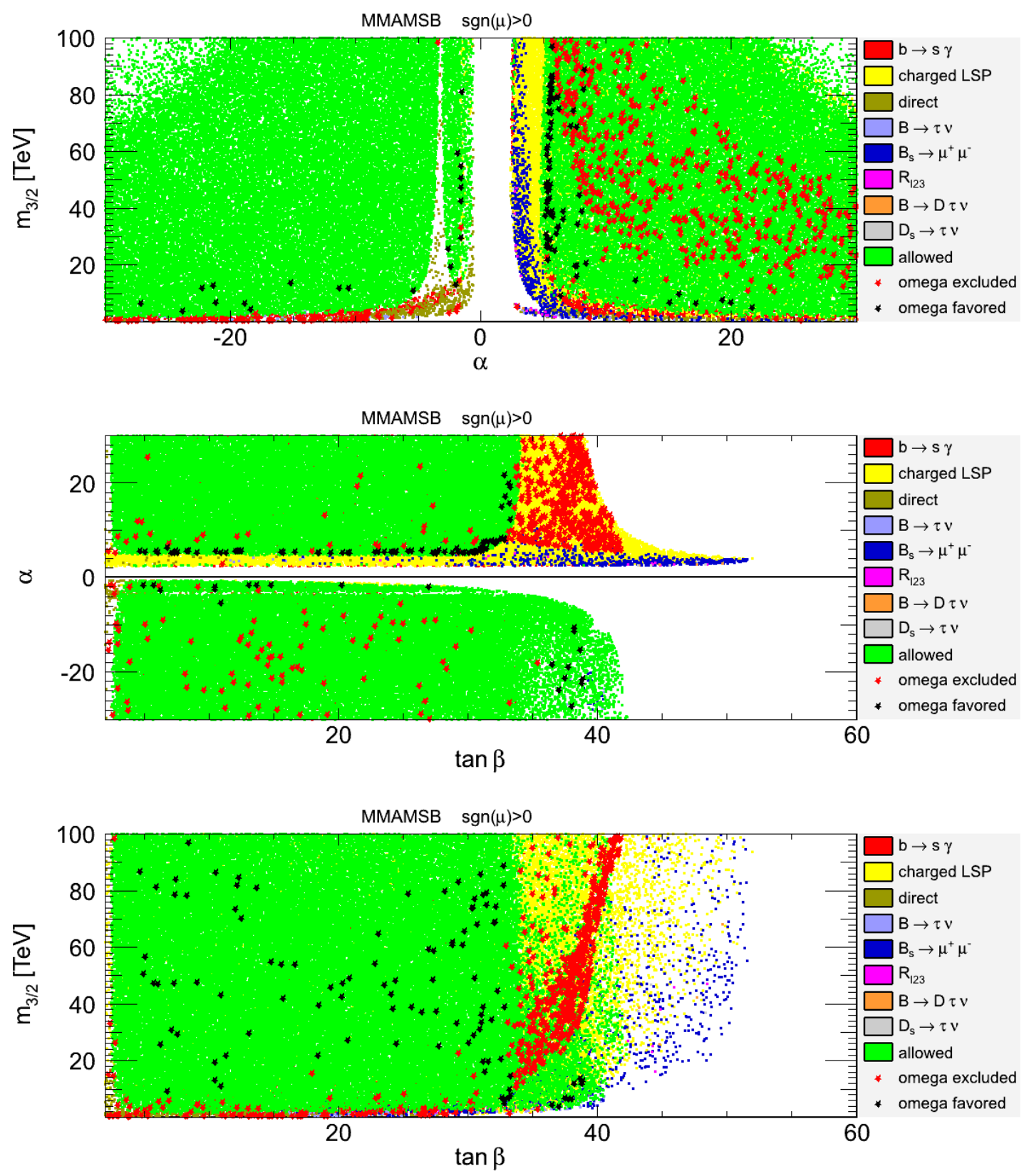

Figure 5. Constraints on the MMAMSB parameter space. The colour codes are the same as in figure 1.

The singlet field mass parameter can also be replaced by the singlet expectation value $v_{s}$. Expanding the singlet field $S$ around $v_{s}$ gives rise to an effective parameter $\mu_{\mathrm{eff}}=\lambda v_{s}$.

One can also define an effective doublet mass such as

$$
m_{A}^{2} \equiv \frac{\lambda v_{s}}{\sin \beta \cos \beta}\left(A_{\lambda}+\kappa v_{s}\right) .
$$

Once the MSSM-like parameters (and in particular $\mu_{\text {eff }}$ ) have been fixed by the specification of the AMSB scenario, we are left with four additional independent NMSSM-specific 

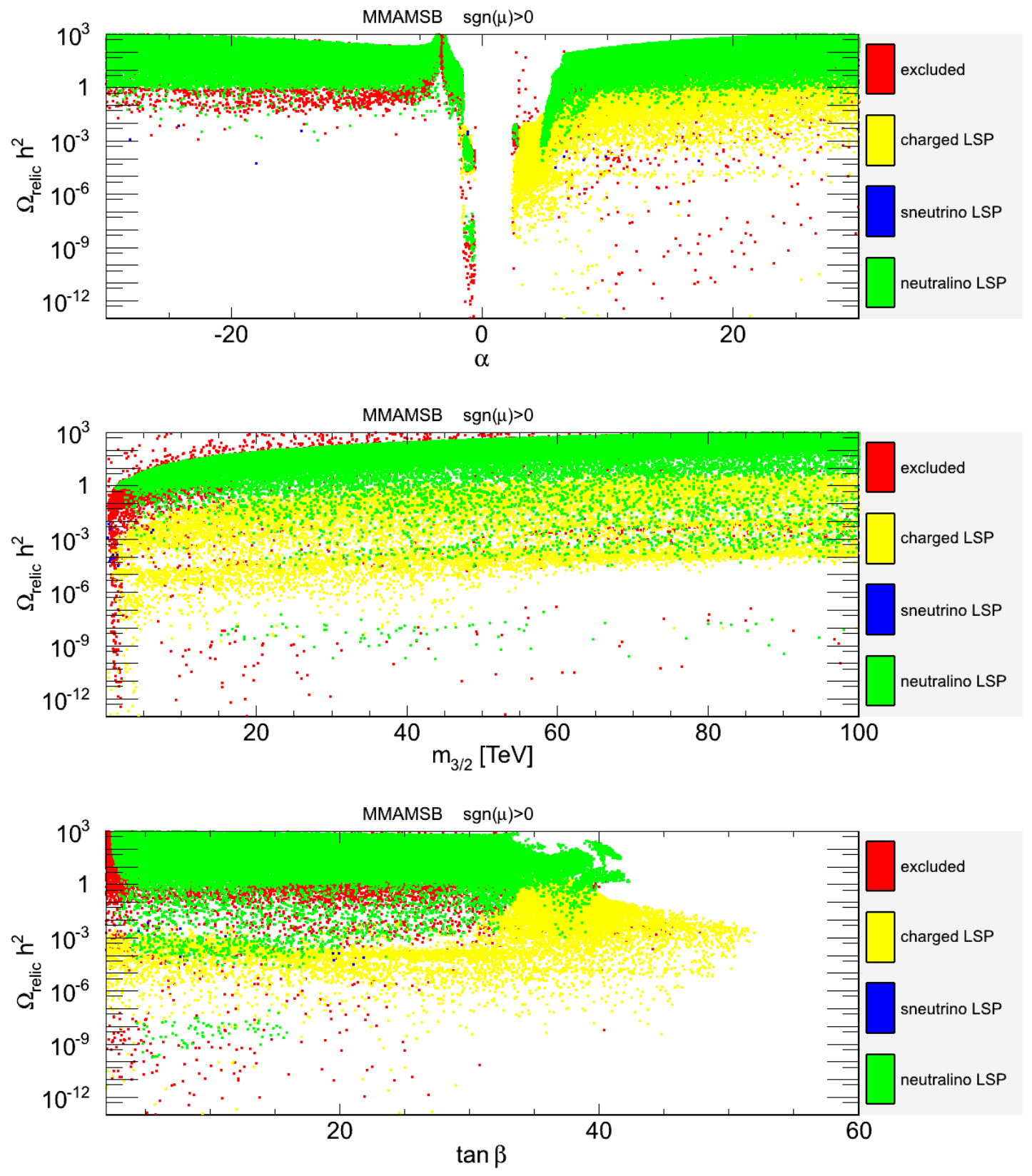

Figure 6. Relic density in function of the MMAMSB parameters. The colour codes are the same as in figure 2 .

parameters:

$$
\lambda, \quad \kappa, A_{\kappa}, M_{A}
$$

We scan over the intervals $\lambda \in[-0.7,0.7] \mathrm{GeV}, \kappa \in[-0.7,0.7], A_{\kappa} \in[-2000,2000] \mathrm{GeV}$ and $M_{A} \in[5,1000]$. We review in the following the differences between the different AMSB scenarios when applied to the NMSSM. 


\section{1 mNAMSB}

Minimal NMSSM-AMSB (mNAMSB) parameter points are generated here using NMSSMTools 2.3.4 [28], and flavour constraints and relic density are computed with SuperIso Relic v3.0 [14-18].

In figure 7 , the parameter space of mNAMSB is presented. As in figure 1, no point satisfy simultaneous flavour, direct and relic density constraints. The direct limits are less constraining than in the MSSM, but the flavour constraints are stronger and exclude a larger part of the NAMSB parameter space in comparison with AMSB.

Figure 8 reveals more differences between the mAMSB and mNAMSB models. First, the zone not excluded by flavour and direct constraints having a relic density around $10^{-9}$ does not exist in the NAMSB, a new green zone appears for $m_{3 / 2}>60$, and its relic density around $10^{-2}$ is much closer to the WMAP constraint. However, as in the mAMSB scenario, the mNAMSB model is globally disfavoured by the standard cosmology.

\section{$3.2 \quad$ NHCAMSB}

We generate the NMSSM-HCAMSB (NHCAMSB) parameter points using NMSSMTools 2.3.4 [28]. The obtained constraints are shown in figure 9. Again, we see that no parameter point satisfies at the same time the relic density constraint and the direct and flavour constraints. Similarly to the mNAMSB scenario, NHCAMSB is less constrained by the direct mass limits, but is more excluded by the flavour constraints.

Figure 10 shows the relic density in function of the different parameters. The green region that exists in the HCAMSB scenario for a relic density around $10^{-8}$ disappears in the NHCAMSB, and a new region opens up around $10^{-2}$. However, as the mNAMSB scenario, the NHCAMSB scenario remains also disfavoured by the standard cosmology.

\subsection{NMMAMSB}

The NMSSM-MMAMSB (NMMAMSB) scenario leads to similar results as the MMAMSB scenario. As can be seen in figure 11, there exists many points satisfying all the constraints, including relic density, especially for values of $\alpha$ near to 0 .

Figure 12 reveals a difference, as the calculated relic density takes values between $10^{-4}$ and $10^{3}$, which is more restrictive in comparison to the MSSM case.

\section{AMSB and relic density in alternative cosmology}

We have seen in the previous section that the relic density imposes severe constraints to the parameter spaces, excluding a major part of the AMSB scenarios. However, the relic density calculation is generally based on the simplistic standard model of cosmology. In this section, we reinterpret the previous results by considering four different alternatives to the cosmological standard scenario. For this study, we focus our interest on six different benchmark points, which are described in table 1 and are representative of the allowed parameter space in the different models. The mass spectra associated to these points are shown in figure 13. 

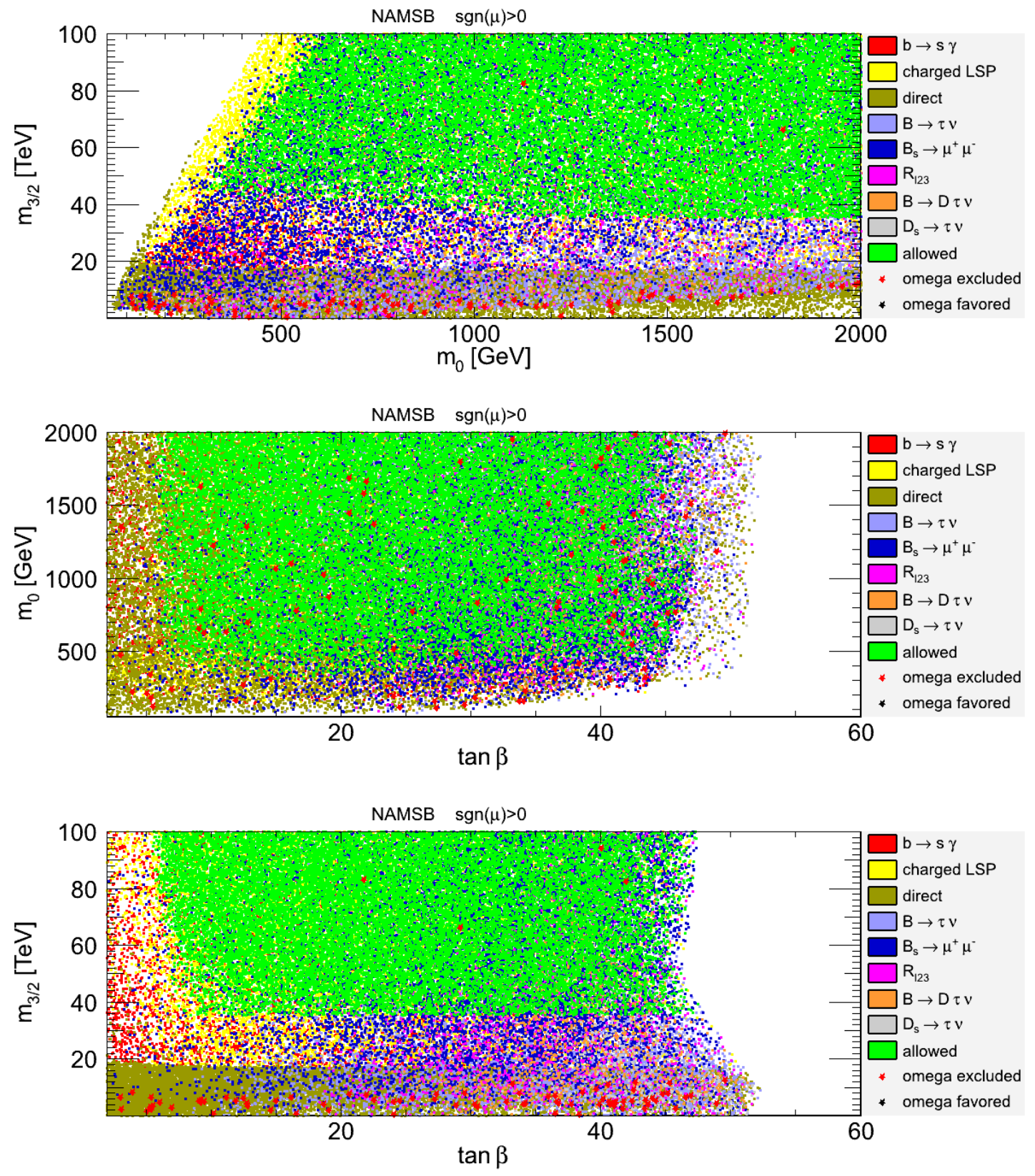

Figure 7. Constraints on the mNAMSB parameter space. The colour codes are the same as in figure 1.

\subsection{Alternative cosmological scenarios}

In the following, we consider that dark matter is composed of exclusively one particle produced thermally.

The density number of supersymmetric particles is determined by the Boltzmann equation $[29,30]$ :

$$
\frac{d n}{d t}=-3 H n-\langle\sigma v\rangle\left(n^{2}-n_{e q}^{2}\right)
$$



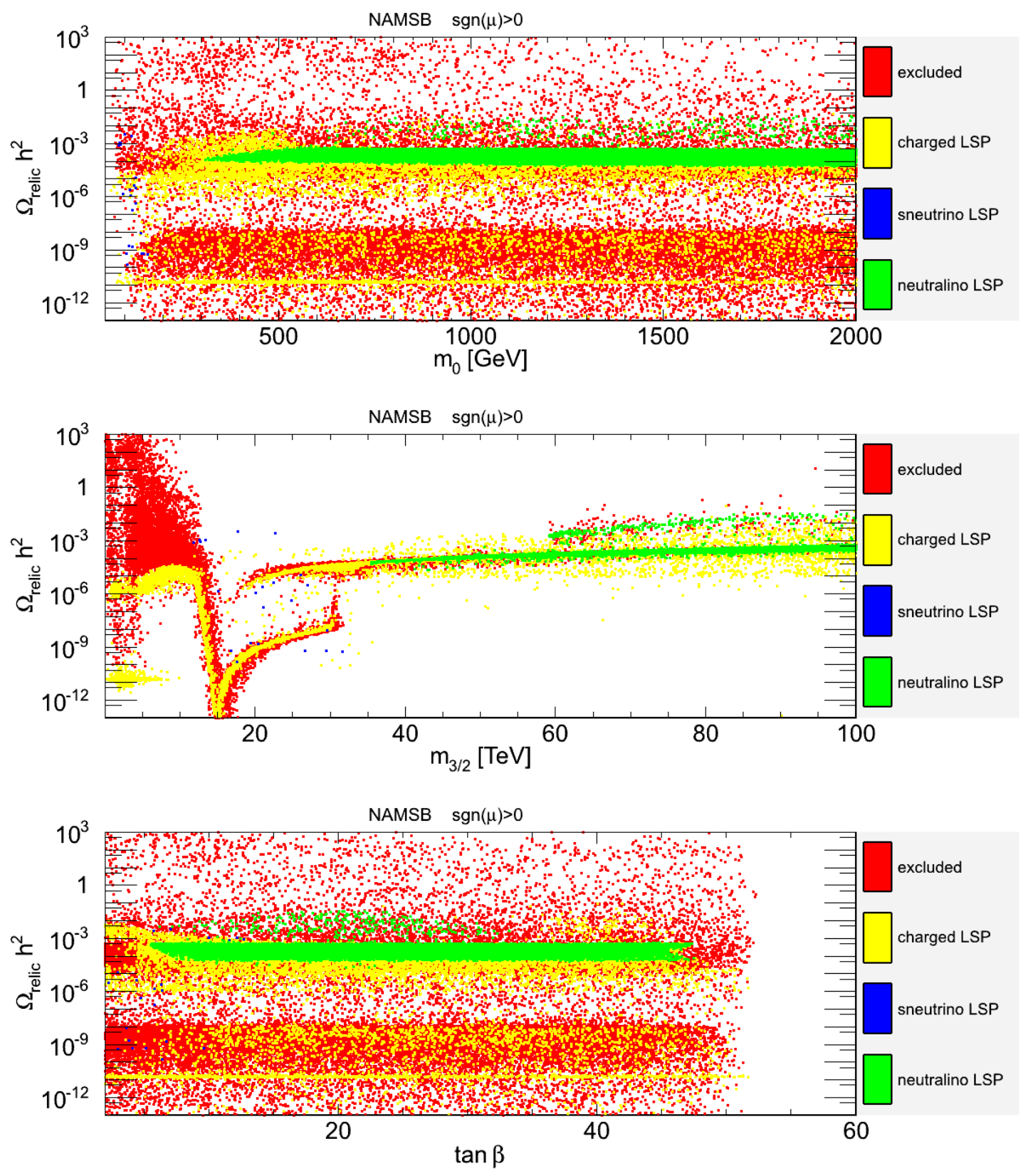

Figure 8. Relic density in function of the mNAMSB parameters. The colour codes are the same as in figure 2 .

where $n$ is the number density of supersymmetric particles, $\langle\sigma v\rangle$ is the thermally averaged annihilation cross-section, $H$ is the Hubble parameter, $n_{e q}$ is the relic particle equilibrium number density. The expansion rate $H$ is determined by the Friedmann equation:

$$
H^{2}=\frac{8 \pi G}{3} \rho_{\text {rad }}
$$



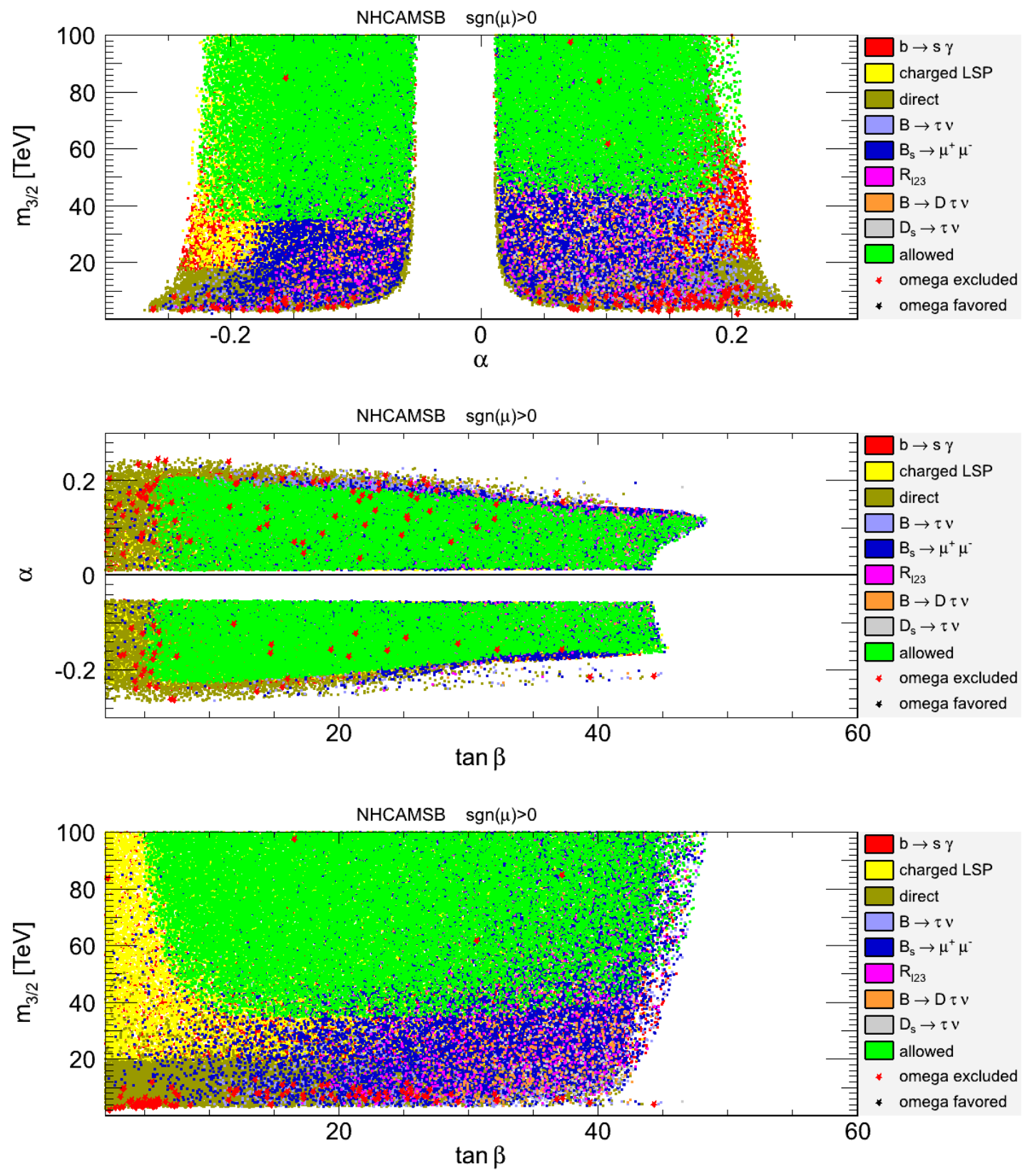

Figure 9. Constraints on the NHCAMSB parameter space. The colour codes are the same as in figure 1.

where $\rho$ is the total energy density of the Universe. The entropy evolution reads:

$$
\frac{d s}{d t}=-3 H s,
$$

where $s$ is the total entropy density. Solving and evolving simultaneously eqs. (4.1), (4.2) and (4.3) enable to compute the relic density in our present Universe. In the standard cosmology, the dominant component before Big-Bang Nucleosynthesis is considered to be radiation, which is constituted of all relativistic particles. This assumption is however 

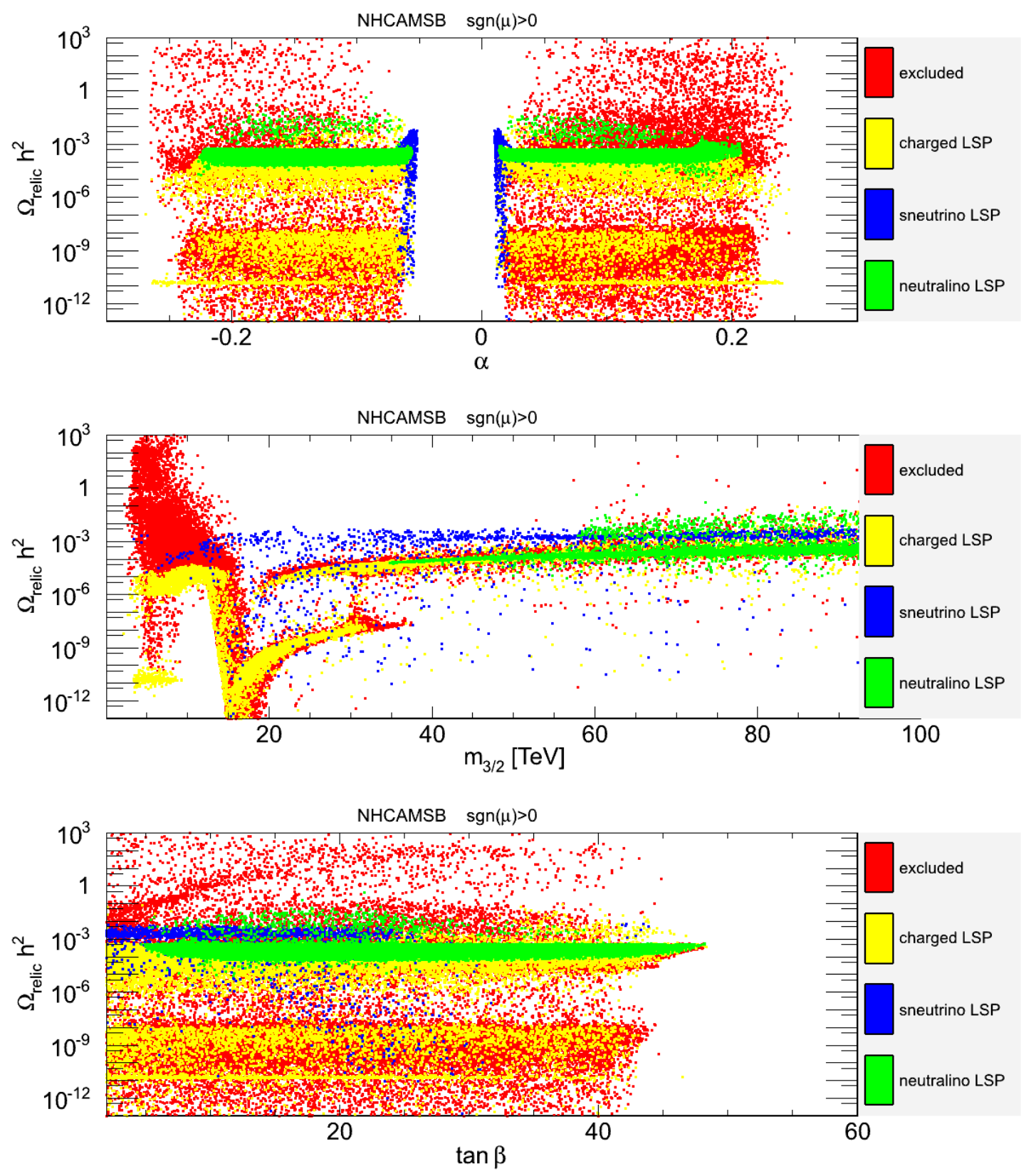

Figure 10. Relic density in function of the NHCAMSB parameters. The colour codes are the same as in figure 2 .

relaxed in many cosmological scenarios. The last two equations can indeed be written more generally as $[2,3]$

$$
\begin{aligned}
H^{2} & =\frac{8 \pi G}{3}\left(\rho_{\text {rad }}+\rho_{D}\right), \\
\frac{d s}{d t} & =-3 H s+\Sigma_{D},
\end{aligned}
$$

$\rho_{D}$ parametrises a modified evolution of the total density of the Universe, beyond radiation 

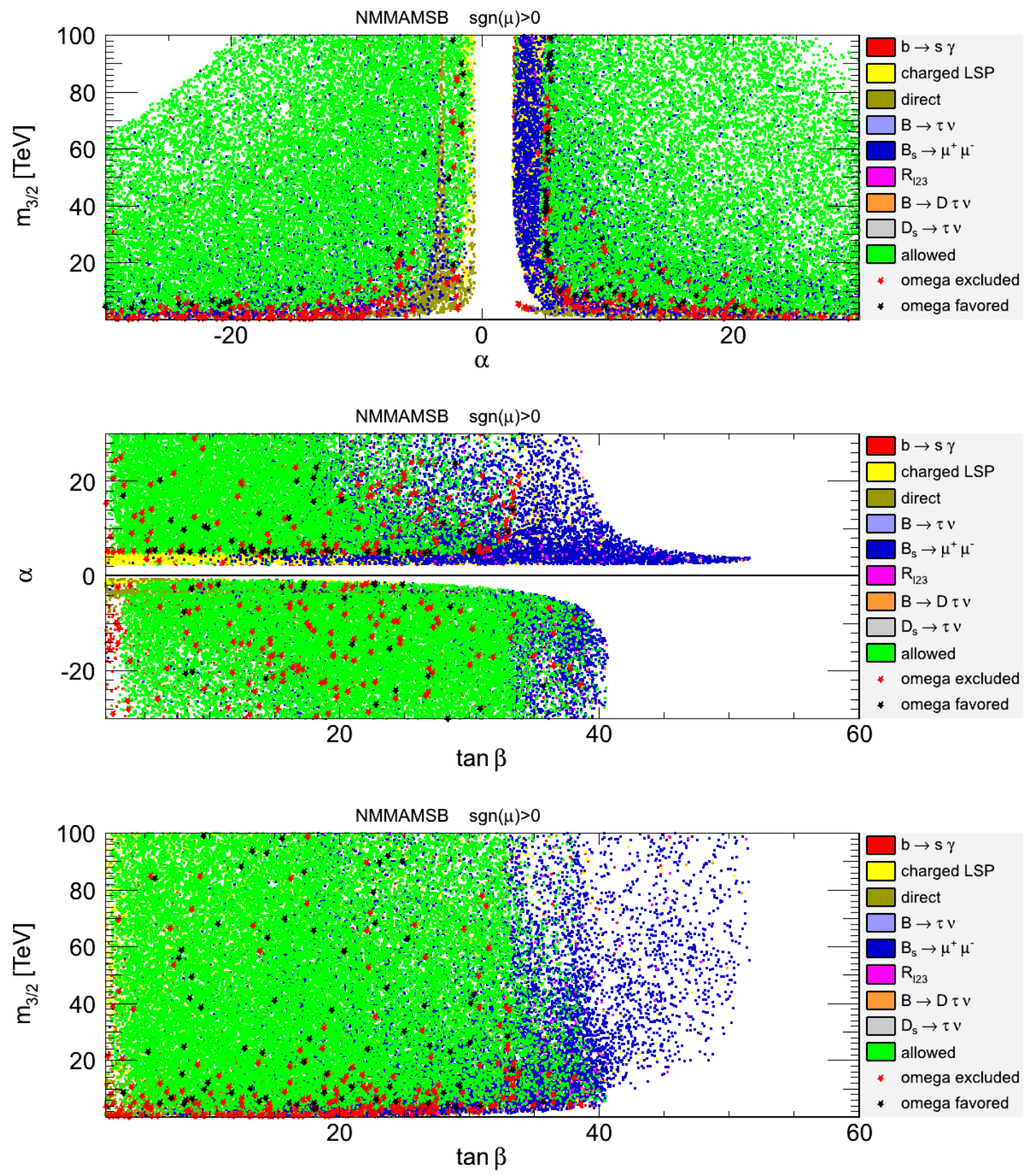

Figure 11. Constraints on the NMMAMSB parameter space. The colour codes are the same as in figure 1.

density $\rho_{\text {rad }} . \Sigma_{D}$ parametrises here effective entropy fluctuations due to unknown properties of the Early Universe. The radiation energy and entropy density evolutions are known and can be written as usual:

$$
\rho_{\text {rad }}=g_{\mathrm{eff}}(T) \frac{\pi^{2}}{30} T^{4}, \quad s_{\text {rad }}=h_{\mathrm{eff}}(T) \frac{2 \pi^{2}}{45} T^{3} .
$$

In the following, we consider two scenarios in which the energy density is modified, and two scenarios with a modified entropy content. 

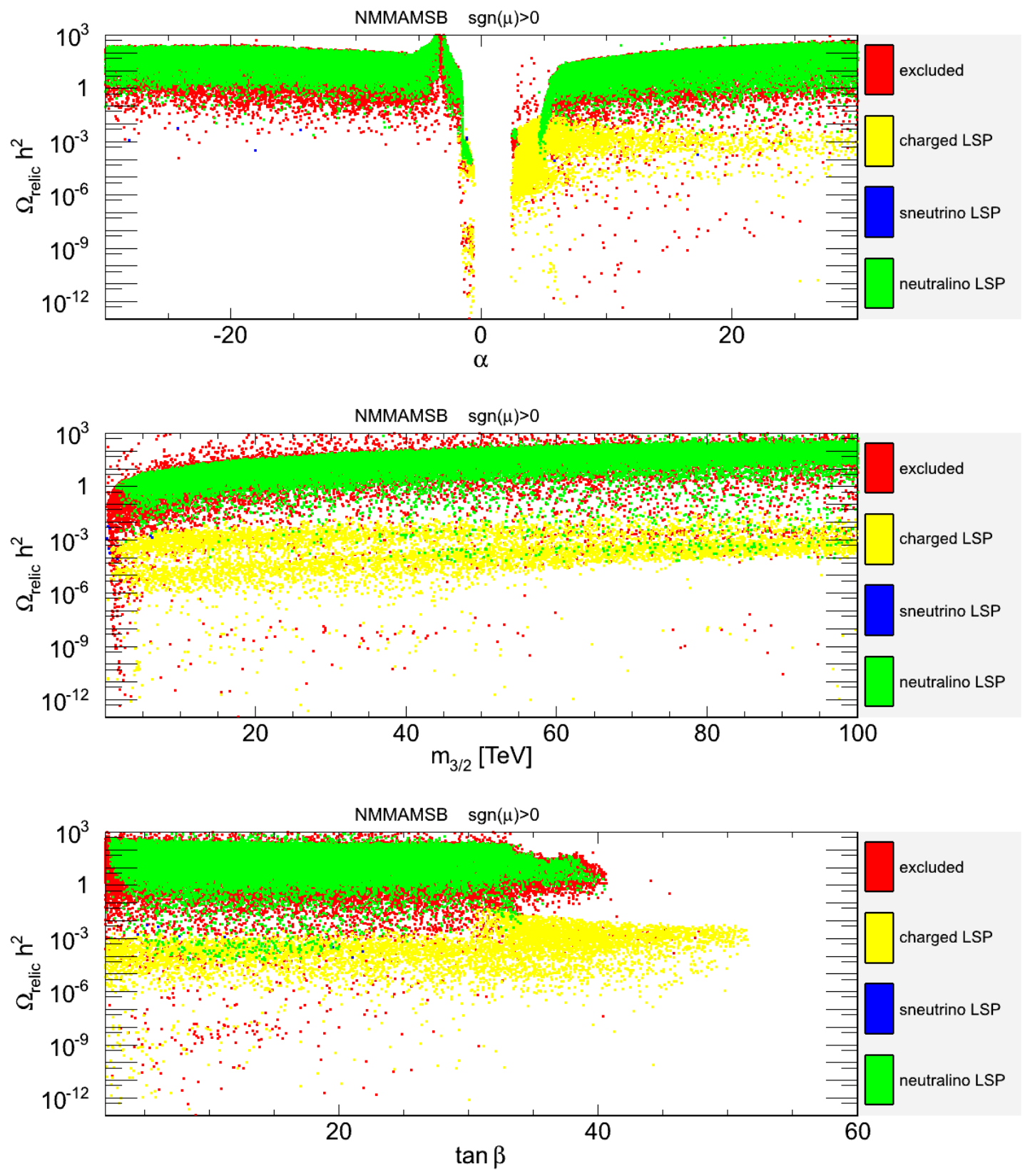

Figure 12. Relic density as a function of the NMMAMSB parameters. The colour codes are the same as in figure 2 .

\subsection{Quintessence}

The quintessence model is one of the most well-known models for dark energy. It is based on a cosmological scalar field which has presently a negative constant pressure $P$ and a positive constant energy density $\rho$ such as $P \approx-\rho$ [31]. This behaviour is achieved when the kinetic term of the scalar field equilibrates the potential. However, in the early Universe, the scalar field has a dominating kinetic term, leading to a positive pressure such as $P \approx \rho$. During this period, the energy density was varying very quickly, such as $\rho \propto T^{6}$. 


\begin{tabular}{|c|c|c|c|c|c|c|c|}
\hline Point & Model & $\Omega_{D M} h^{2}$ & $m_{0}(\mathrm{GeV})$ & $\alpha$ & $m_{3 / 2}(\mathrm{TeV})$ & $\tan \beta$ & $M_{A}(\mathrm{GeV})$ \\
\hline A & mAMSB & $3.33 \times 10^{-4}$ & 1000 & $\mathrm{n} / \mathrm{a}$ & 80 & 30 & 1060.5 \\
\hline B & mAMSB & $4.63 \times 10^{-10}$ & 2000 & $\mathrm{n} / \mathrm{a}$ & 20 & 40 & 1322.8 \\
\hline C & HCAMSB & $3.24 \times 10^{-4}$ & $\mathrm{n} / \mathrm{a}$ & 0.1 & 80 & 10 & 1931.3 \\
\hline D & MMAMSB & 5.98 & $\mathrm{n} / \mathrm{a}$ & 10 & 20 & 30 & 1904.4 \\
\hline E & MMAMSB & $6.95 \times 10^{2}$ & $\mathrm{n} / \mathrm{a}$ & 20 & 100 & 10 & 2320.5 \\
\hline F & mNAMSB & $1.21 \times 10^{-2}$ & 1300 & $\mathrm{n} / \mathrm{a}$ & 70 & 20 & 770 \\
\hline
\end{tabular}

Table 1. Benchmark points for testing alternative cosmology scenarios. All these points have $\mu>0$ and are in agreement with all the flavour and direct search constraints but would be excluded by WMAP constraints based on the standard cosmology. For the point F extra parameters are needed to specify a point in the parameter space, which are chosen to be: $\lambda=-0.1, \kappa=0.5$ and $A_{\kappa}=1500 \mathrm{GeV}$.

We study here a quintessence scenario in which the quintessence field before Big-Bang Nucleosynthesis was dominating the expansion of the Universe. In this case [1]:

$$
\rho_{D}(T)=\kappa_{\rho} \rho_{r a d}\left(T_{B B N}\right)\left(\frac{T}{T_{B B N}}\right)^{6}
$$

where $\kappa_{\rho}$ is the proportion of quintessence to radiation at the BBN temperature $(\sim 1 \mathrm{MeV})$. We consider that $\kappa_{\rho}$ is a free parameter, which can be constrained using the BBN abundance constraints. To compute the abundance of the elements produced during the primordial nucleosynthesis, we use the code AlterBBN [32] integrated into SuperIso Relic. Comparing the abundances to the observational constraints, we obtain limits on $\kappa_{\rho}$.

\subsection{Late decaying inflaton}

The second scenario we consider here is a late decay of an inflaton field. The inflaton field is a scalar field which is considered to be responsible for the rapid inflation of the early Universe. Generally, the inflaton is considered to decay into standard particles much before the relic decoupling from the primordial soup. However, several models evoke the possibility of a late decay of the inflaton, around the time of BBN. From [33, 34], there exist cosmological models in which the late decay of a scalar field reheats the Universe to a low reheating temperature, which can be smaller than the freeze-out temperature, without spoiling primordial nucleosynthesis. The decay of this scalar field into radiation increases the entropy and modifies the expansion rate of the Universe. We consider here such a scenario in which we neglect the eventual entropy production, and we takes [1]

$$
\rho_{D}(T)=\kappa_{\rho} \rho_{\text {rad }}\left(T_{B B N}\right)\left(\frac{T}{T_{B B N}}\right)^{8} .
$$

The exponent is here increased from 6 to 8 in comparison to the quintessence field, as mentioned also in $[35,36]$. Such a modification of the expansion rate can be also achieved in a Universe with extra-dimensions modifying the Friedmann equations [37]. 
Point A

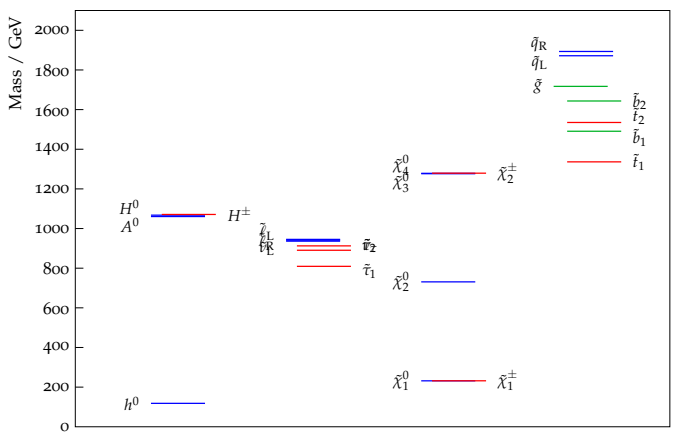

Point C

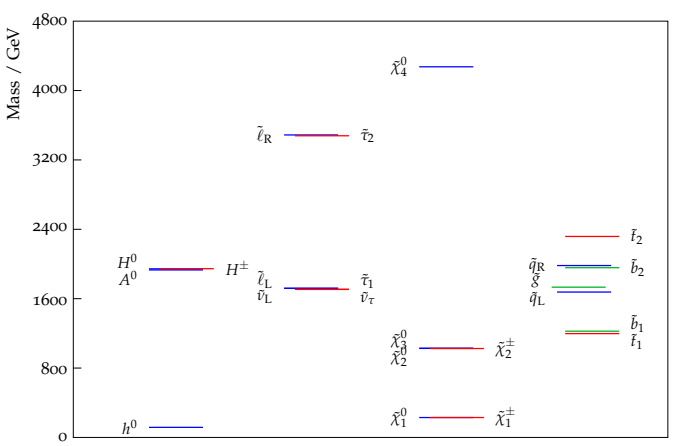

Point E

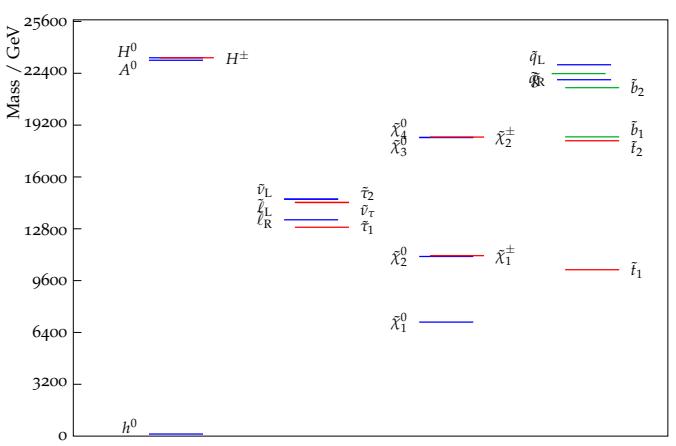

Point B

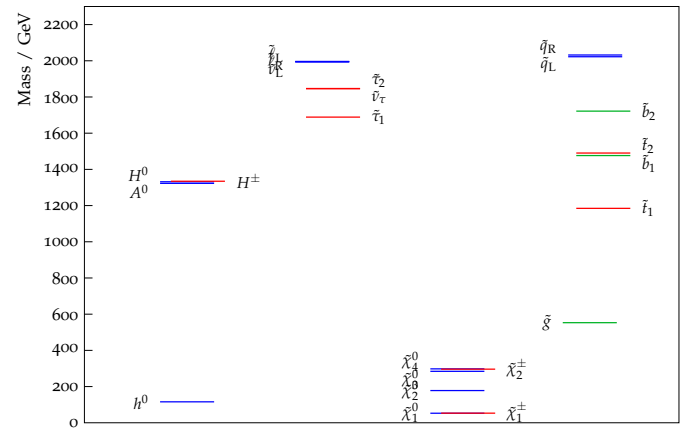

Point D

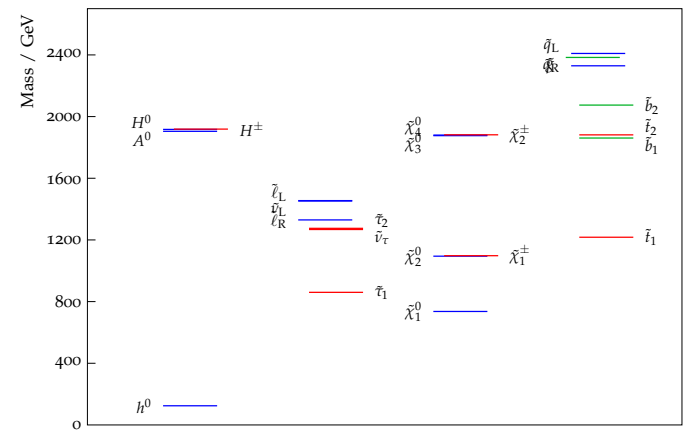

Point F

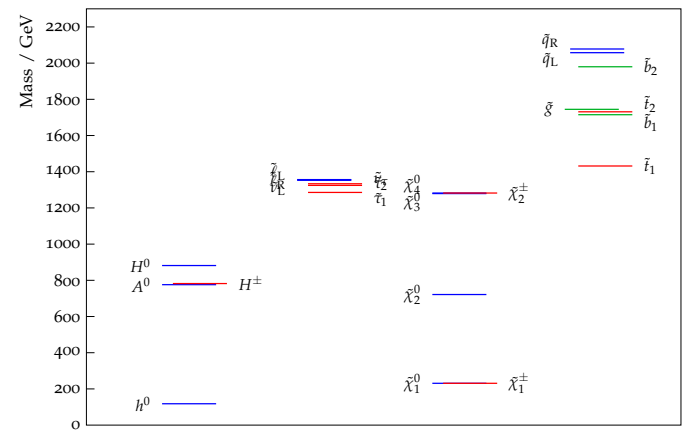

Figure 13. Mass spectra of the six benchmark points. Note that the scales are not identical for all spectra.

\subsection{Primordial entropy production}

In this third scenario, we assume that a primordial entropy production due to an unknown component occurs. In general, such an entropy production should be accompanied with energy production, but to better estimate the deviation of the relic density in such a cosmological scenario, we neglect here the energy production, and we consider that the Universe has, in addition to the standard radiation entropy density, a dark entropy density 
evolving like $[2,3]$

$$
s_{D}(T)=\kappa_{s} s_{r a d}\left(T_{B B N}\right)\left(\frac{T}{T_{B B N}}\right)^{3},
$$

where $\kappa_{s}$ is the ratio of effective dark entropy density over radiation entropy density at the time of BBN. The corresponding entropy production is related to $s_{D}$ by the relation

$$
\Sigma_{D}=\sqrt{\frac{4 \pi^{3} G}{5}} \sqrt{1+\tilde{\rho}_{D}} T^{2}\left[\sqrt{g_{\mathrm{eff}}} s_{D}-\frac{1}{3} \frac{h_{\mathrm{eff}}}{g_{*}^{1 / 2}} T \frac{d s_{D}}{d T}\right],
$$

with

$$
g_{*}^{1 / 2}=\frac{h_{\mathrm{eff}}}{\sqrt{g_{\mathrm{eff}}}}\left(1+\frac{T}{3 h_{\mathrm{eff}}} \frac{d h_{\mathrm{eff}}}{d T}\right)
$$

and

$$
\tilde{\rho}_{D}=\frac{\rho_{D}}{\rho_{\text {rad }}}
$$

\subsection{Late reheating}

In this last scenario, the reheating temperature $T_{R H}$ is smaller than the neutralino freeze out temperature $\left(T_{\text {f.o. }} \simeq m_{\chi} / 20 \mathrm{GeV}\right)$ [34], $T_{R H}$ should be considered as a cosmological parameter that can take any value around a few $\mathrm{MeV}$ and then the neutralinos decoupled from the plasma before the end of the reheating process so their relic density will differ from its standard value. We consider that the inflaton decays around the time of BigBang nucleosynthesis, generating entropy. From the standard late reheating scenarios, we assume that the entropy production evolves like $[2,3]$

$$
\Sigma_{D}(T)=\kappa_{\Sigma} \Sigma_{r a d}\left(T_{B B N}\right)\left(\frac{T_{B B N}}{T}\right)
$$

for $T>1 \mathrm{MeV}$ and that this entropy production stops at the time of BBN. We again neglect the energy production or non-thermal production of particles in order to better understand the effects of a reheating entropy production on the relic density. In term of entropy density, we have

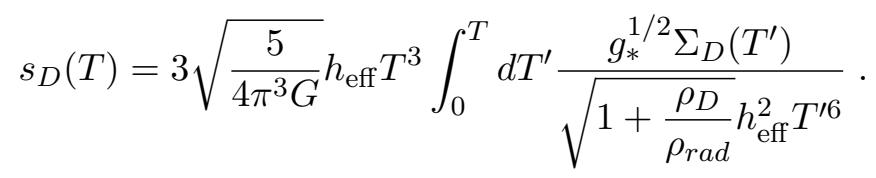

\subsection{BBN constraints and modified relic density}

The different scenarios do not have impact on the cosmological observations, but they can modify the abundance of the elements. We compute with AlterBBN [32] the abundance of the elements in the different scenarios for each benchmark points, varying the single parameter for a given scenario, and we apply the following conservative constraints [38]:

$$
\begin{aligned}
& 0.240<Y_{p}<0.258, \quad 1.2 \times 10^{-5}<{ }^{2} H / H<5.3 \times 10^{-5}, \\
& 0.57<{ }^{3} \mathrm{H} /{ }^{2} \mathrm{H}<1.52, \quad{ }^{7} \mathrm{Li} / \mathrm{H}>0.85 \times 10^{-10}, \quad{ }^{6} \mathrm{Li} /{ }^{7} \mathrm{Li}<0.66,
\end{aligned}
$$



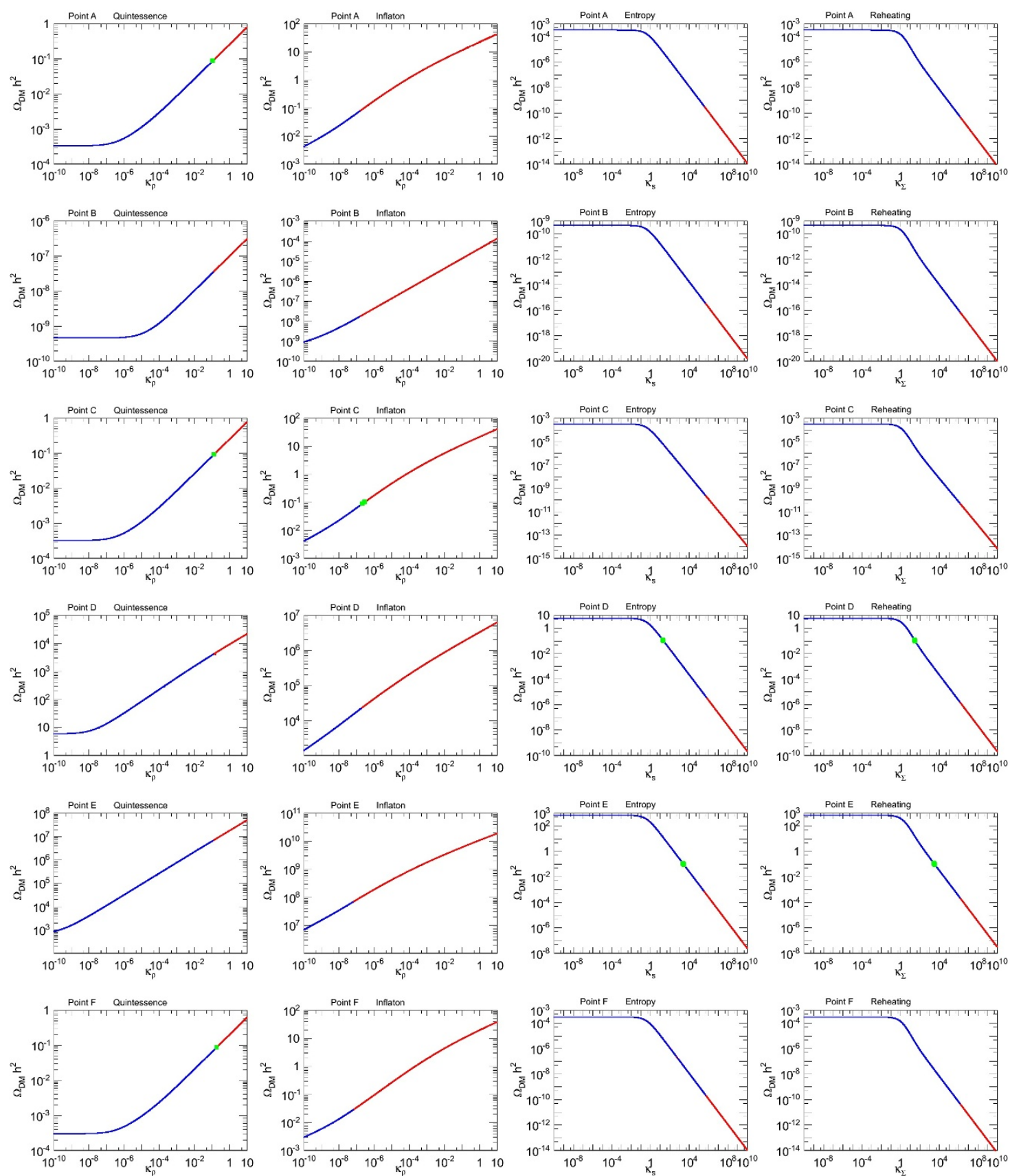

Figure 14. Relic density in function of the cosmological model parameters. The red lines correspond to points excluded by the BBN constraints in eq. (4.15), whereas the blue lines correspond to a region with a correct abundance of the elements. The green squares correspond to points allowed by the BBN constraints and giving a relic density in agreement with the WMAP dark matter interval.

for the helium abundance $Y_{p}$ and the primordial ${ }^{2} \mathrm{H} / \mathrm{H},{ }^{3} \mathrm{H} /{ }^{2} \mathrm{H},{ }^{7} \mathrm{Li} / \mathrm{H}$ and ${ }^{6} \mathrm{Li} /{ }^{7} \mathrm{Li}$ ratios.

In figure 14, we consider the relic density calculated for each of the benchmark points (A-F, from top to bottom) and for different cosmological scenarios (from left to right). 
These plots reveal that the quintessence and inflaton scenarios globally increase the relic density, while the entropy and reheating scenarios decrease it. The comparison with the BBN constraints is also represented, and the red part of the curves is excluded at 95\% C.L., while the blue part gives a correct abundance of the elements. As general features, the quintessence and inflaton scenarios can increase the relic density by three orders of magnitudes without interfering with the BBN constraints, and the entropy and reheating scenarios can decreased to a factor of $10^{6}$. Therefore, apart from point B which has an extremely low relic density value in the standard cosmological scenario, all the other benchmark points can have a relic density value compatible with the cosmological observations if a non minimal cosmological scenario is considered.

\section{Generalised relic density constraints}

We have shown with different well-known cosmological scenarios, that the relic density constraints can be very strongly relaxed. Therefore, we propose to compare the relic density calculated in the standard model of cosmology to the following interval

$$
10^{-4}<\Omega_{D M} h^{2}<10^{5}
$$

to take into consideration the fact that it is possible to increase any relic density calculated in the standard cosmology by three orders of magnitude, and to decrease it by six orders, with non-standard cosmological scenarios in agreement with the current cosmological data. We can re-apply the relic density constraints, and the results are shown in figures 15-20. As in the figures of section 2, the green zones correspond to regions in agreement with all flavour and direct constraints, but not necessarily with the relic density constraint. We added in the figures black points, which correspond to regions also in agreement with the new dark matter interval. It is clear that the allowed regions are therefore much larger than with the initial relic density interval, but a surprising result is that even with the very large interval we use here for the relic density, the relic density constraint still excludes large part of the parameter spaces. In particular, in the mAMSB and HCAMSB scenarios and their NMSSM counterparts, the relic density constraints clearly exclude the region $m_{3 / 2} \lesssim 40 \mathrm{TeV}$. The MMAMSB scenario however is not constrained anymore when using the new dark matter interval.

\section{LHC phenomenology}

The benchmark points selected for testing alternative cosmology scenarios are in agreement with precision flavour and direct search constraints. It turns out that the phenomenology expected at the LHC is quite peculiar. The mass spectra for the benchmark points we considered show that the lightest neutralino is the LSP and the lightest chargino is in most cases very close in mass to the neutralino (points A, B, C, F). This will give rise to peculiar signatures due to the very limited number of open channels for the decay modes. We analyse in more detail in the following each of the six benchmark points previously 

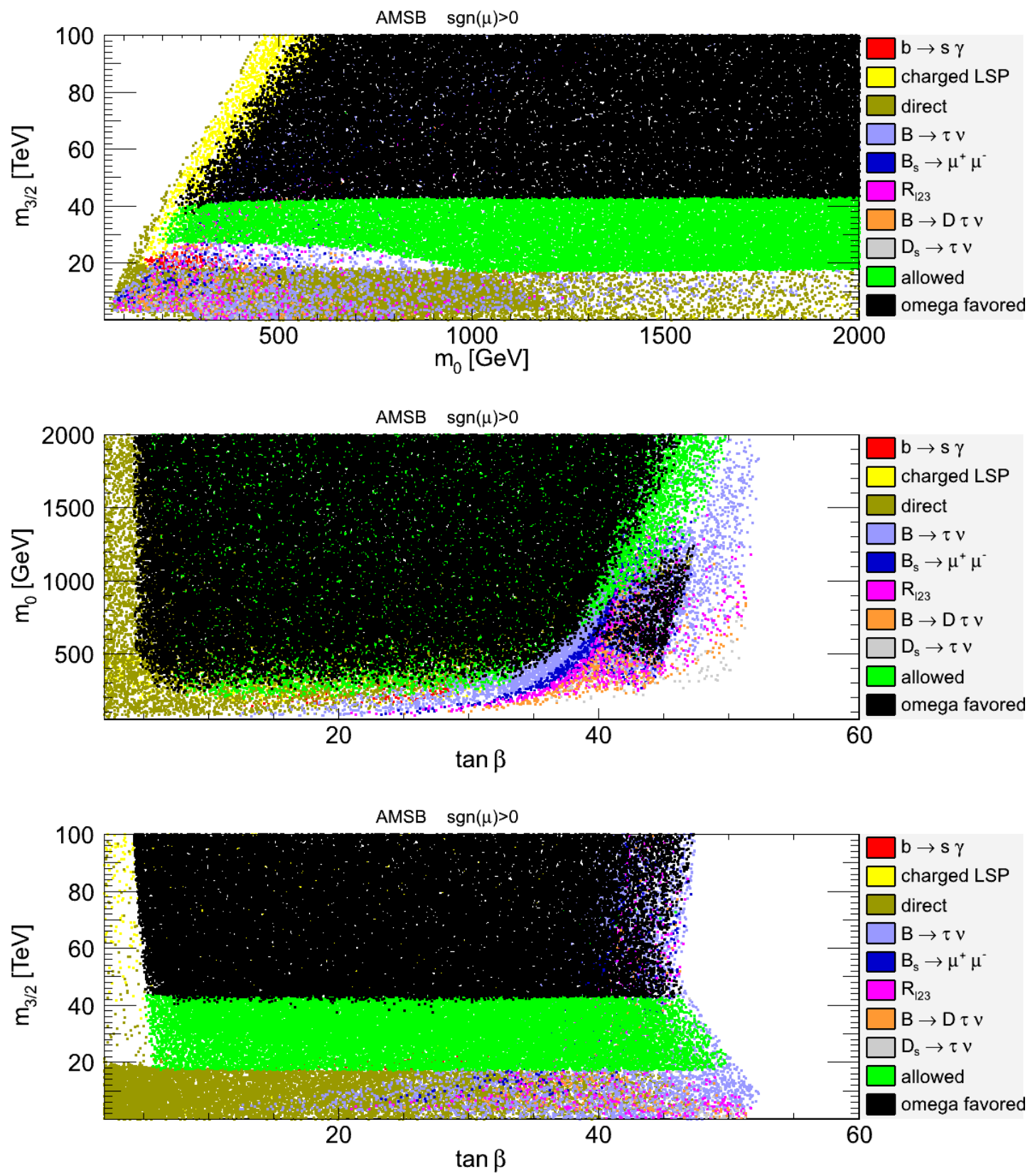

Figure 15. Constraints on the minimal AMSB parameter space. The exclusion regions are plotted in the order given in the legend. The red zones are excluded by the inclusive branching ratio of $B \rightarrow X_{s} \gamma$, the yellow ones correspond to charged LSP, the olive green area is excluded by direct collider constraints, the light blue zones are excluded by $\operatorname{BR}(B \rightarrow \tau \nu)$, the dark blue zones by $\operatorname{BR}\left(B_{s} \rightarrow \mu^{+} \mu^{-}\right)$, the magenta zones by $R_{\ell 23}$, the orange zones by $\operatorname{BR}(B \rightarrow D \tau \nu)$ and the grey zones by $\operatorname{BR}\left(D_{s} \rightarrow \tau \nu\right)$. The green areas are in agreement with all the previously mentioned constraints. The black area corresponds to parameters in agreement with all constraints, including the revised relic density interval given in eq. (5.1). 

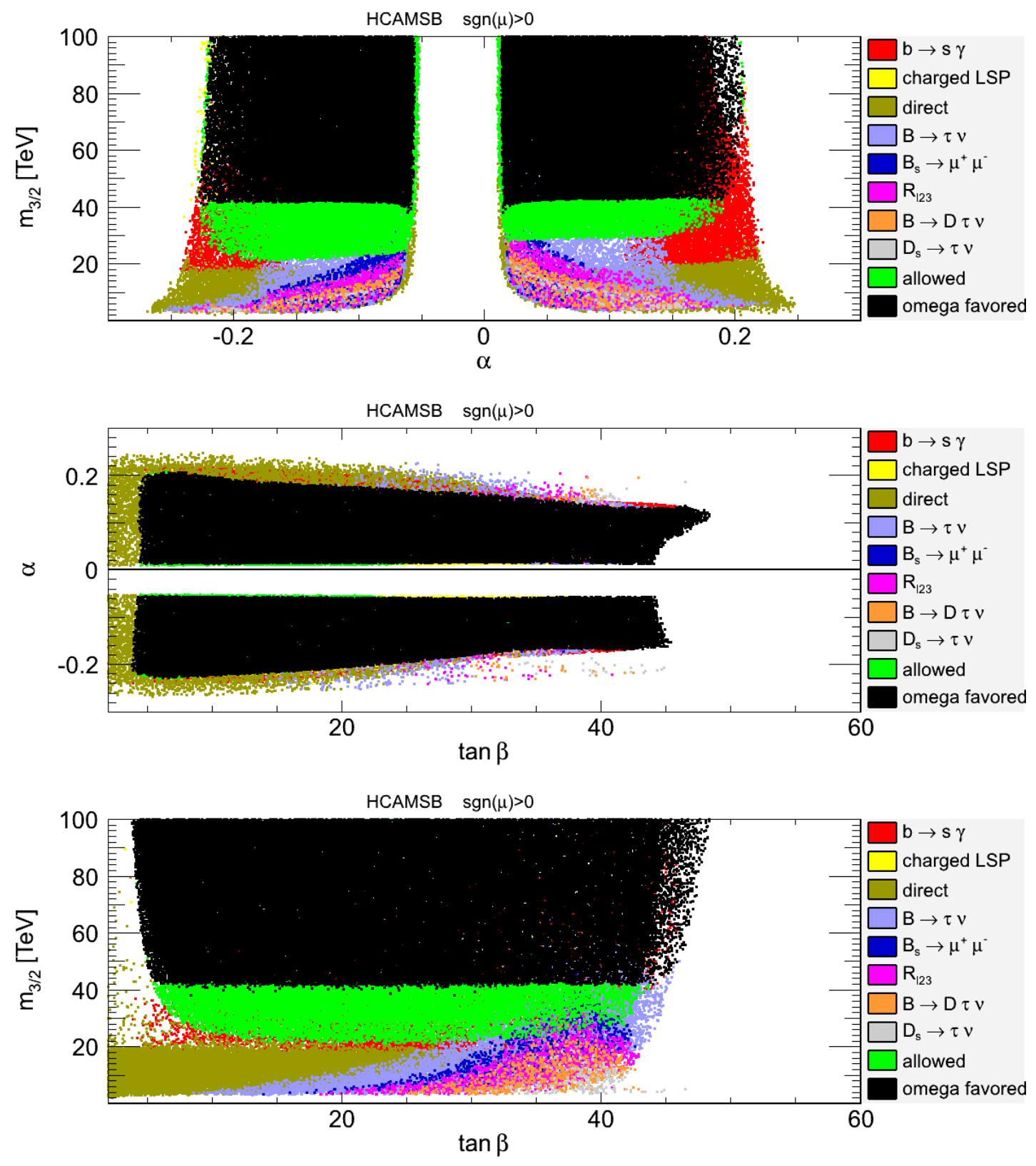

Figure 16. Constraints on the HCAMSB parameter space with the revised relic density interval given in eq. (5.1). The colour codes are the same as in figure 15.

selected. Production of charginos and neutralinos takes place at the LHC via cascade decays of squark and gluinos and via the direct production channels

$$
p p \rightarrow \tilde{\chi}_{i} \tilde{\chi}_{j}+X
$$

where the s-channel exchange of an off-shell $\mathrm{W}$ or $\mathrm{Z}$ or photon, and the contribution of SUSY-QCD diagrams are important. Indeed these cross-sections receive important SUSYQCD corrections, typical values are given in [39] or can be obtained using a Monte Carlo program including the relevant $\mathrm{K}$-factors or the detailed next-to-leading matrix elements. 

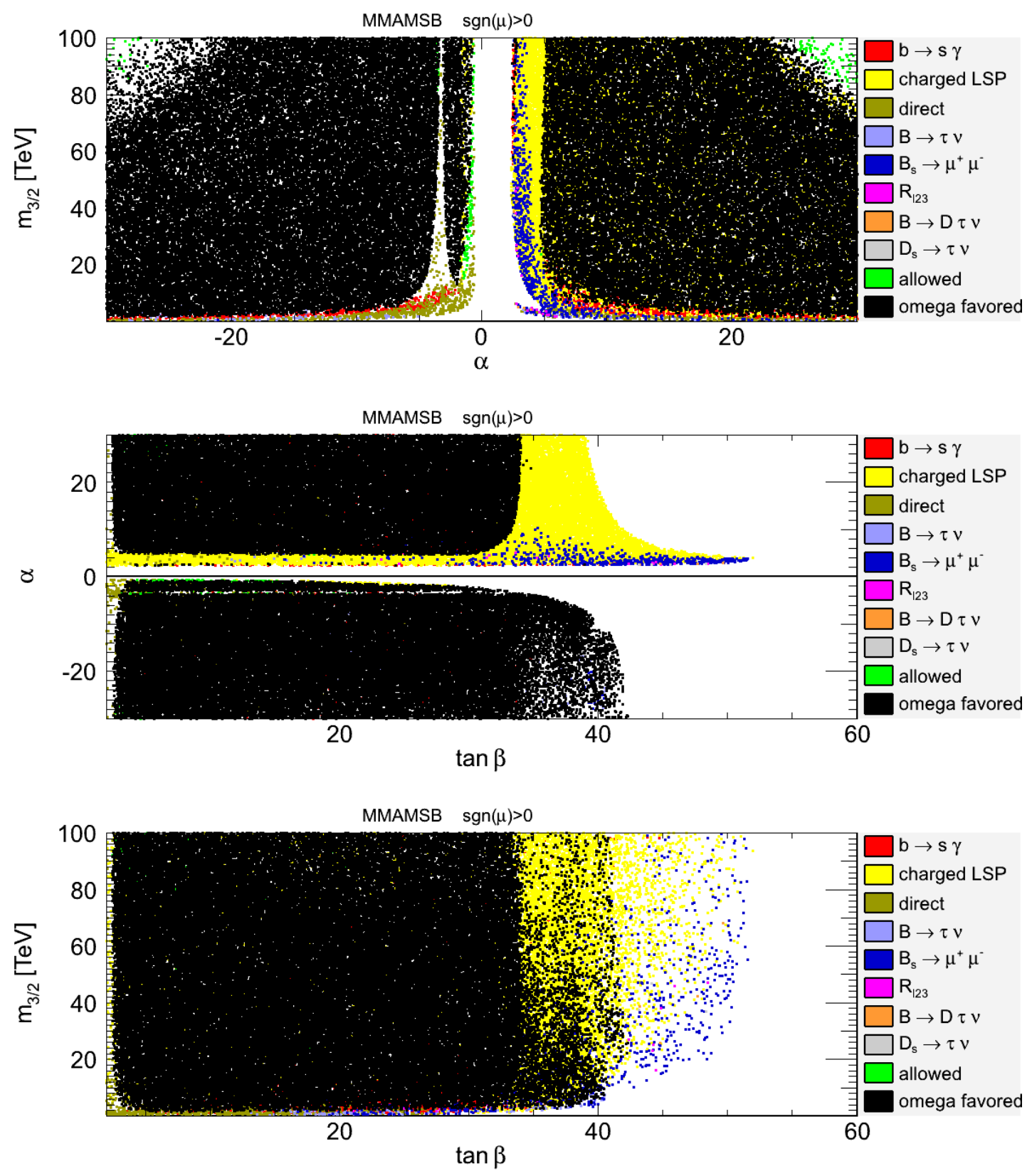

Figure 17. Constraints on the MMAMSB parameter space with the revised relic density interval given in eq. (5.1). The colour codes are the same as in figure 15.

\section{1 mAMSB point A}

The minimal AMSB scenario is disfavoured by the standard cosmology as very far from the WMAP dark matter allowed interval. The region allowed in our more general analysis typically favours points in which the lightest chargino and neutralino are very close in mass and not so heavy. For point A, $m_{\tilde{\chi}_{1}^{0}}=231.76 \mathrm{GeV}$ and $m_{\tilde{\chi}_{1}^{+}}=231.93 \mathrm{GeV}$ so that the mass splitting is only $170 \mathrm{MeV}$. Due to this small mass splitting, the open decay modes for the 

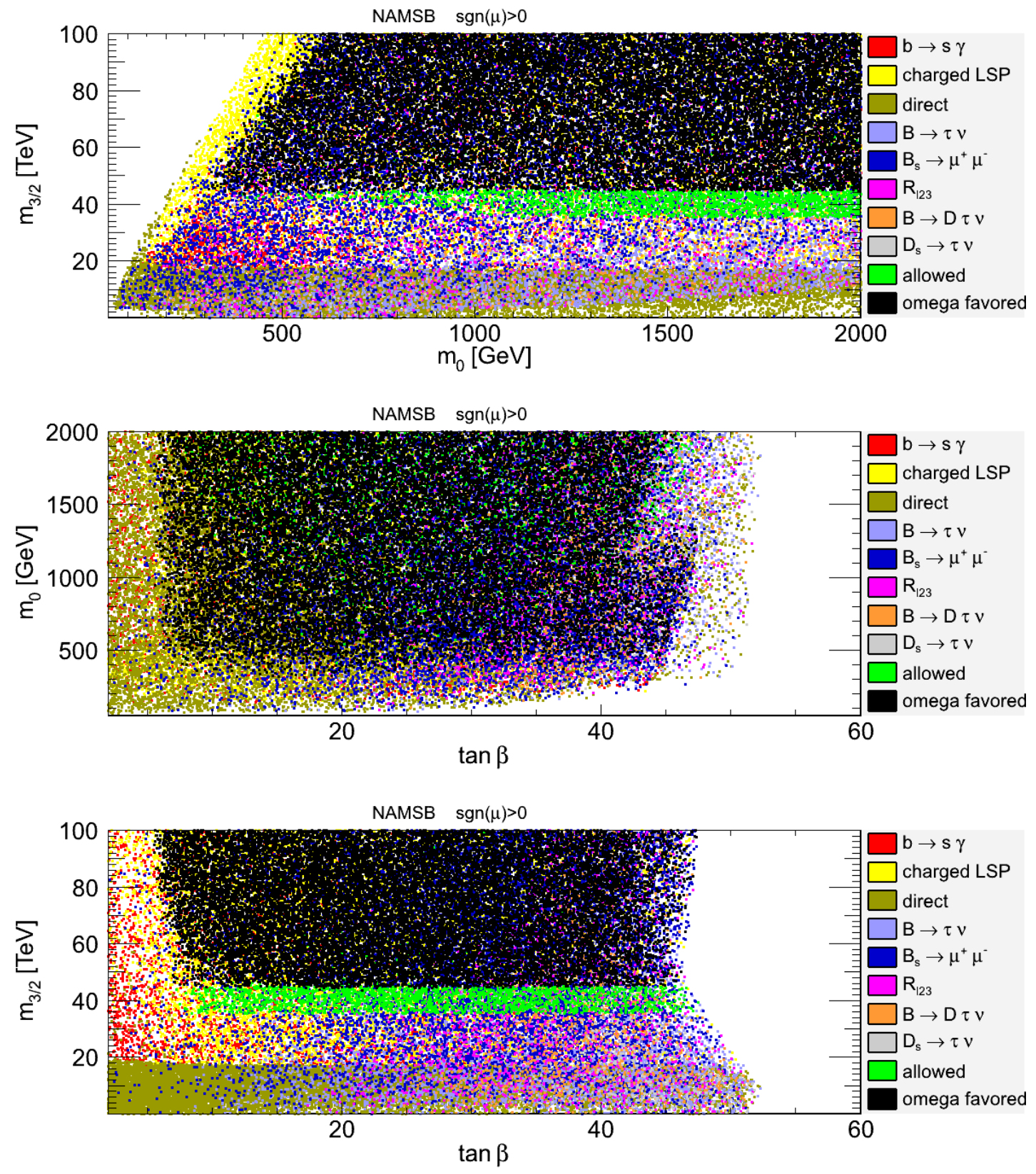

Figure 18. Constraints on the mNAMSB parameter space with the revised relic density interval given in eq. (5.1). The colour codes are the same as in figure 15.

$\tilde{\chi}_{1}^{+}$are (neglecting very small modes below the $10^{-3}$ level) $\tilde{\chi}_{1}^{+} \rightarrow \tilde{\chi}_{1}^{0} l \nu$, where

$$
\begin{aligned}
\operatorname{BR}\left(\tilde{\chi}_{1}^{+} \rightarrow \tilde{\chi}_{1}^{0} \mu^{+} \nu_{\mu}\right) & \simeq 1.87 \times 10^{-2} \\
\operatorname{BR}\left(\tilde{\chi}_{1}^{+} \rightarrow \tilde{\chi}_{1}^{0} e^{+} \nu_{e}\right) & \simeq 1.87 \times 10^{-2} \\
\operatorname{BR}\left(\tilde{\chi}_{1}^{+} \rightarrow \tilde{\chi}_{1}^{0} \pi^{+} \rightarrow \tilde{\chi}_{1}^{0} e^{+} \nu_{e}\right) & \simeq 0.96
\end{aligned}
$$

so that $l=e$ and $l=\mu$ have the same branching through the three body decays while most of the signal with $\tilde{\chi}_{1}^{0} e^{+} \nu_{e}$ is through the two body production of a charged pion. The next 

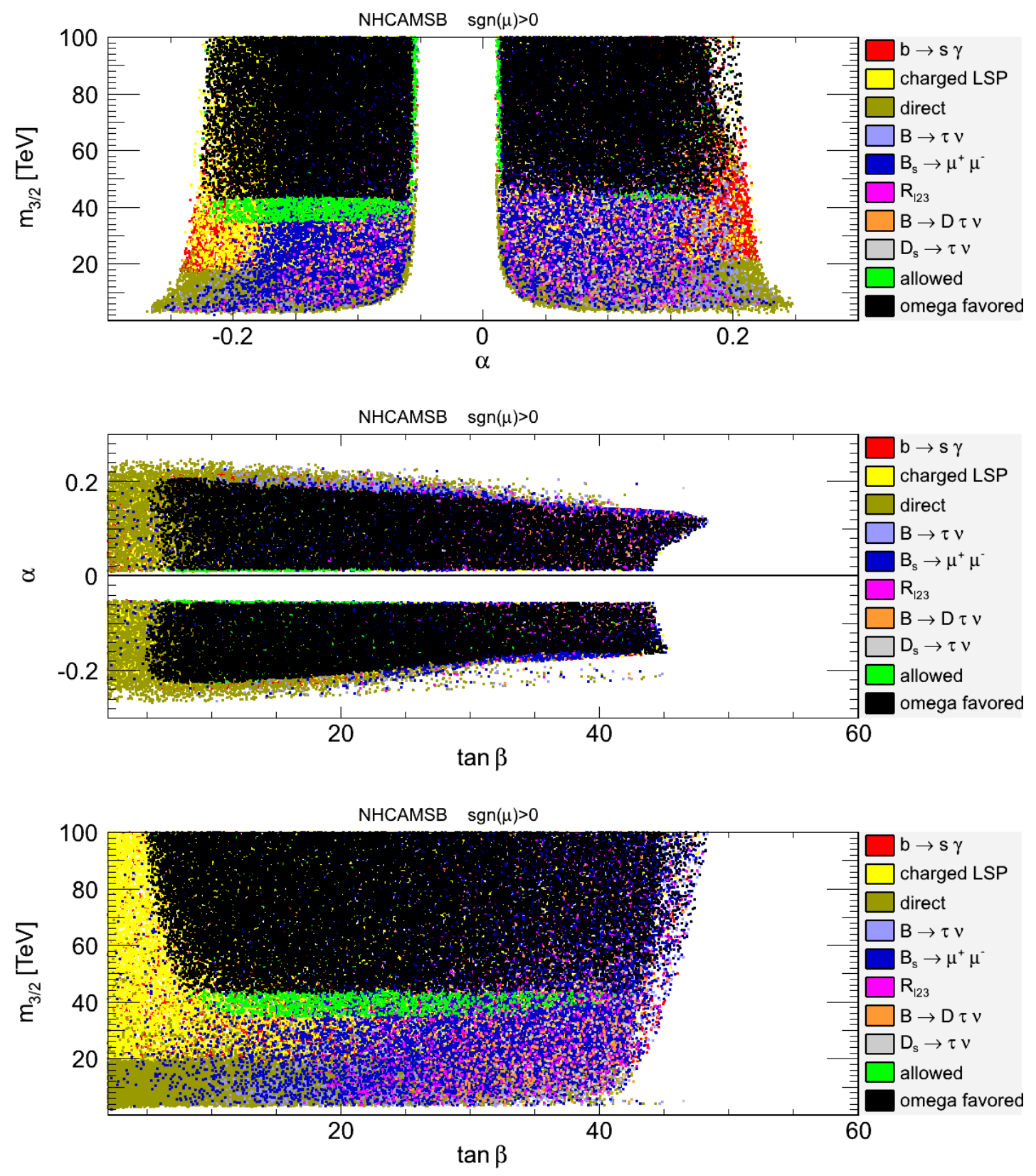

Figure 19. Constraints on the NHCAMSB parameter space with the revised relic density interval given in eq. (5.1). The colour codes are the same as in figure 15.

lightest particle is the $\tilde{\chi}_{2}^{0}$, decaying mainly to $\tilde{\chi}_{1}^{+}$and $\mathrm{W}$ or $\tilde{\chi}_{1}^{0}$ and SM-like Higgs as for the light Higgs for this point $(118.15 \mathrm{GeV})$ the decay is kinematically allowed:

$$
\begin{aligned}
\operatorname{BR}\left(\tilde{\chi}_{2}^{0} \rightarrow \tilde{\chi}_{1}^{ \pm} W^{\mp}\right) & \simeq 0.75 \\
\operatorname{BR}\left(\tilde{\chi}_{2}^{0} \rightarrow \tilde{\chi}_{1}^{0} h^{0}\right) & \simeq 0.19 \\
\operatorname{BR}\left(\tilde{\chi}_{2}^{0} \rightarrow \tilde{\chi}_{1}^{0} l^{+} l^{-}\right) & \simeq 5.5 \times 10^{-3},
\end{aligned}
$$



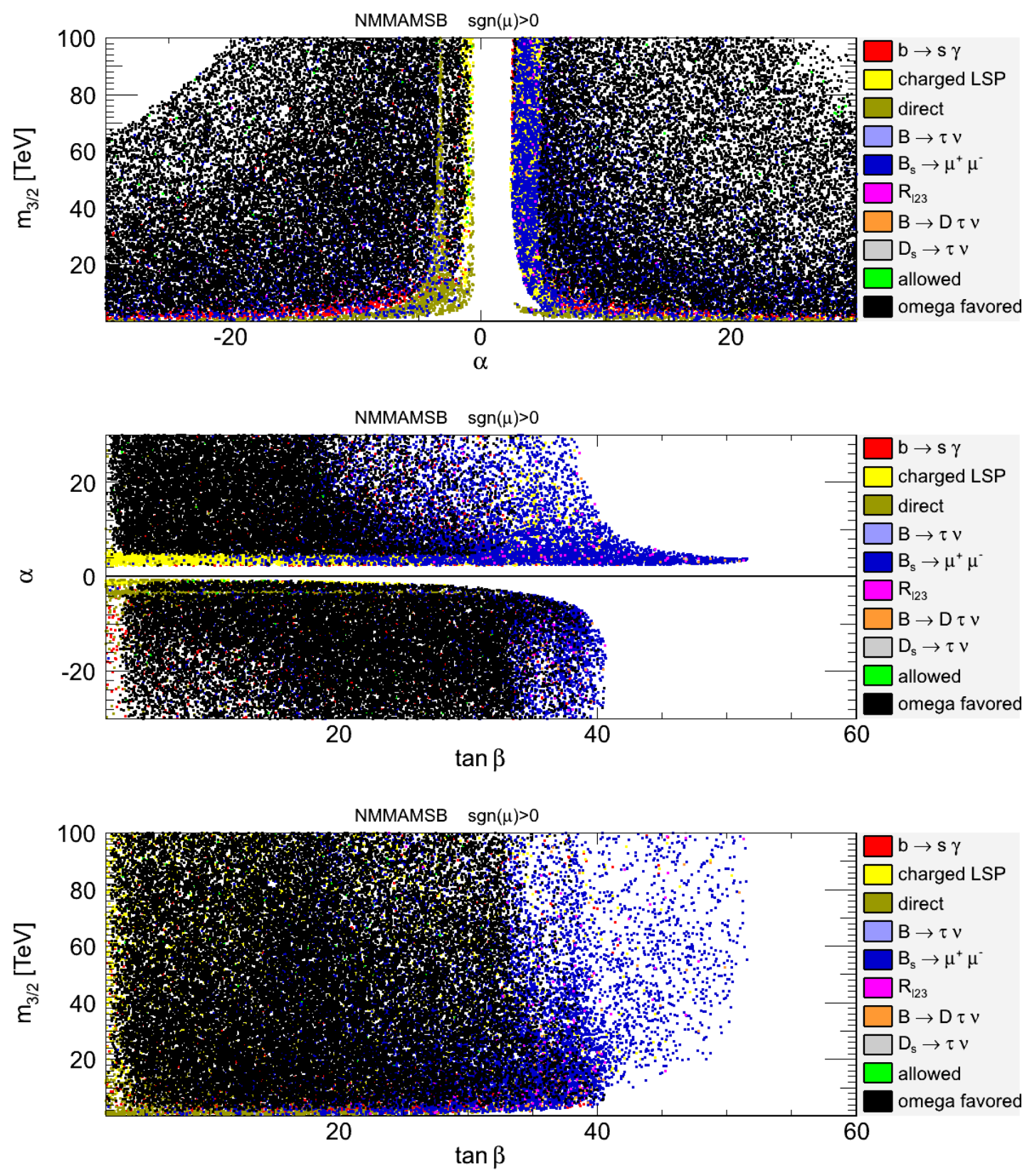

Figure 20. Constraints on the NMMAMSB parameter space including the revised relic density interval. The colour codes are the same as in figure 15 .

where $l=e, \mu$. Since the branching of the mode $\tilde{\chi}_{1}^{ \pm} \rightarrow \tilde{\chi}_{1}^{0} l^{ \pm} \nu$ is $100 \%$ and the mode $\tilde{\chi}_{2}^{0} \rightarrow \tilde{\chi}_{1}^{0} l^{+} l^{-}$is non-negligible one can study the clean trilepton signal usually suggested at hadron colliders [40, 41].

\section{2 mAMSB point $B$}

The benchmark point B is in the minimal AMSB scenario too. The situation is similar to the previous point with in this case the lightest chargino and neutralino lighter than the 
SM-like Higgs boson. For point B, $m_{\tilde{\chi}_{1}^{0}}=52.70 \mathrm{GeV}$ and $m_{\tilde{\chi}_{1}^{+}}=53.07 \mathrm{GeV}$ so that the mass splitting is only $63 \mathrm{MeV}$. The decay modes for the lightest chargino are similar to the previous case

$$
\begin{aligned}
\operatorname{BR}\left(\tilde{\chi}_{1}^{+} \rightarrow \tilde{\chi}_{1}^{0} \mu^{+} \nu_{\mu}\right) & \simeq 5.26 \times 10^{-2} \\
\operatorname{BR}\left(\tilde{\chi}_{1}^{+} \rightarrow \tilde{\chi}_{1}^{0} e^{+} \nu_{e}\right) & \simeq 5.26 \times 10^{-2} \\
\operatorname{BR}\left(\tilde{\chi}_{1}^{+} \rightarrow \tilde{\chi}_{1}^{0} \pi^{+} \rightarrow \tilde{\chi}_{1}^{0} e^{+} \nu_{e}\right) & \simeq 0.89,
\end{aligned}
$$

and for the second neutralino

$$
\begin{aligned}
\operatorname{BR}\left(\tilde{\chi}_{2}^{0} \rightarrow \tilde{\chi}_{1}^{ \pm} W^{\mp}\right) & \simeq 0.8 \\
\operatorname{BR}\left(\tilde{\chi}_{2}^{0} \rightarrow \tilde{\chi}_{1}^{0} h^{0}\right) & \simeq 0.1 \\
\operatorname{BR}\left(\tilde{\chi}_{2}^{0} \rightarrow \tilde{\chi}_{1}^{0} Z^{0}\right) & \simeq 9.6 \times 10^{-2} .
\end{aligned}
$$

The SM-Higgs boson (with a mass of $115.7 \mathrm{GeV}$ ) decays with a sizeable branching to pairs of lightest charginos and pairs of lightest neutralinos (branching $1.1 \times 10^{-1}$ and $7.9 \times 10^{-2}$ respectively) while the largest mode is $h^{0} \rightarrow b \bar{b}$ with a branching of $6.5 \times 10^{-1}$.

\subsection{HCAMSB point $\mathrm{C}$}

The HCAMSB scenario is disfavoured by standard cosmology and excluded by the WMAP dark matter allowed interval as for the minimal AMSB scenario discussed above. Also in this case the lightest chargino and neutralino are very close in mass. The masses are $m_{\tilde{\chi}_{1}^{0}}=229.41 \mathrm{GeV}$ and $m_{\tilde{\chi}_{1}^{+}}=229.58 \mathrm{GeV}$ with a splitting of only $17 \mathrm{MeV}$. In this situation the lightest chargino decays are very close to the numbers for point $\mathrm{A}$. The decay modes for the lightest chargino are:

$$
\begin{aligned}
\operatorname{BR}\left(\tilde{\chi}_{1}^{+} \rightarrow \tilde{\chi}_{1}^{0} \mu^{+} \nu_{\mu}\right) & \simeq 1.72 \times 10^{-2} \\
\operatorname{BR}\left(\tilde{\chi}_{1}^{+} \rightarrow \tilde{\chi}_{1}^{0} e^{+} \nu_{e}\right) & \simeq 1.72 \times 10^{-2} \\
\operatorname{BR}\left(\tilde{\chi}_{1}^{+} \rightarrow \tilde{\chi}_{1}^{0} \pi^{+} \rightarrow \tilde{\chi}_{1}^{0} e^{+} \nu_{e}\right) & \simeq 0.96
\end{aligned}
$$

and for the second neutralino

$$
\begin{aligned}
\operatorname{BR}\left(\tilde{\chi}_{2}^{0} \rightarrow \tilde{\chi}_{1}^{ \pm} W^{\mp}\right) & \simeq 0.67 \\
\operatorname{BR}\left(\tilde{\chi}_{2}^{0} \rightarrow \tilde{\chi}_{1}^{0} h^{0}\right) & \simeq 7.3 \times 10^{-2} \\
\operatorname{BR}\left(\tilde{\chi}_{2}^{0} \rightarrow \tilde{\chi}_{1}^{0} Z^{0}\right) & \simeq 0.26
\end{aligned}
$$

In this case the trilepton mode $p p \rightarrow \tilde{\chi}_{1}^{ \pm} \tilde{\chi}_{2}^{0} \rightarrow 3 l+E_{T}$ is especially interesting as the branching fraction $\tilde{\chi}_{1}^{+} \rightarrow \tilde{\chi}_{1}^{0} l \nu$ is $100 \%$ and the one for $\tilde{\chi}_{2}^{0} \rightarrow \tilde{\chi}_{1}^{0} Z^{0}$ is $26 \%$.

\subsection{MMAMSB point D}

The Mixed Modulus AMSB supersymmetry breaking scenario allows for viable dark matter candidates. Benchmark point D has the neutralino LSP with a mass $m_{\tilde{\chi}_{1}^{0}}=736 \mathrm{GeV}$. The next-to-lightest supersymmetric particle is the stau with a mass $m_{\tilde{\tau}}=860 \mathrm{GeV}$ while the 
lightest chargino and the second lightest neutralino are heavier and almost degenerate with a mass of 1095 and $1098 \mathrm{GeV}$ respectively. The stau decays to tau and the lightest neutralino

$$
\operatorname{BR}\left(\tilde{\tau} \rightarrow \tilde{\chi}_{1}^{0} \tau\right)=1
$$

while the lightest chargino decays mainly to

$$
\begin{aligned}
\operatorname{BR}\left(\tilde{\chi}_{1}^{+} \rightarrow \tilde{\tau} \nu_{\tau}\right) & \simeq 0.84 \\
\operatorname{BR}\left(\tilde{\chi}_{1}^{+} \rightarrow \tilde{\chi}_{1}^{0} W\right) & \simeq 0.16 .
\end{aligned}
$$

The second lightest neutralino decays to stau and tau while as the SM-like Higgs is light enough to be produced $(124.3 \mathrm{GeV})$, the decay $\tilde{\chi}_{2}^{0} \rightarrow \tilde{\chi}_{1}^{0} h^{0}$ is also kinematically open

$$
\begin{aligned}
\operatorname{BR}\left(\tilde{\chi}_{2}^{0} \rightarrow \tilde{\tau} \tau\right) & \simeq 0.83 \\
\operatorname{BR}\left(\tilde{\chi}_{2}^{0} \rightarrow \tilde{\chi}_{1}^{0} h^{0}\right) & \simeq 0.16 .
\end{aligned}
$$

\subsection{MMAMSB point $\mathrm{E}$}

For the same model as in the previous paragraph we have also an example of a higher mass spectrum in which all supersymmetric partners are quite heavy with the neutralino LSP above $7 \mathrm{TeV}$ and all other particles above $10 \mathrm{TeV}$.

\section{6 mNAMSB point $\mathrm{F}$}

NMSSM with minimal AMSB scenario is one of the models considered in the previous sections. It is disfavoured by the relic density constraints if standard cosmology is assumed. Point $\mathrm{F}$ is in the allowed zone for flavour, low energy and direct search constraints and is allowed if standard cosmology constraints are relaxed as discussed in the previous sections. From the point of view of LHC searches, this point is similar to point A. For point F, $m_{\tilde{\chi}_{1}^{0}}=230.03 \mathrm{GeV}$ and $m_{\tilde{\chi}_{1}^{+}}=231.02 \mathrm{GeV}$ so that the mass splitting is $99 \mathrm{MeV}$. Apart from these two particles, the next supersymmetric particle (in terms of mass) is the second neutralino (see figure 13) with a mass of $721 \mathrm{GeV}$.

\section{Conclusions and perspectives}

We have considered in details the constraints on different possible realisations of superconformal anomaly mediation breaking mechanisms in supersymmetry. These constraints include the usual LEP, B-factories, Tevatron and LHC searches, but also precision constraints of cosmological origin, namely the WMAP limits on the relic density of cold dark matter. We have discussed the standard cosmological approach and also alternative cosmological scenarios which do not change the cosmological observations but which can affect strongly the constraints on the parameter space of these supersymmetric models based on the relic abundance of dark matter. We therefore show how the dark matter constraints can be weakened in order to avoid strong model dependent assumptions in the choice of the cosmological model. Based on different benchmark points for AMSB models, we performed a detailed analysis of the constraints imposed by particle data and cosmology (both 
standard and alternative) and finally we gave the typical mass spectra and decay modes relevant for the LHC searches. The main lesson that can be learnt in such an exercice is that usual bounds on the parameter space of these models are too restrictive and bear a strong hidden cosmological model dependence in the assumption of the standard cosmological scenario. Concerning the LHC searches, points which are excluded in the standard analysis but permitted in this more general approach, may be quite relevant for testing not only the particle theory models themselves but also alternative cosmological scenarios at the LHC, as in many cases relatively low mass supersymmetric particles are allowed with a peculiar spectrum where the lightest neutralino and chargino are very close in mass.

\section{Acknowledgments}

We would like to thank Nazila Mahmoudi for useful discussions and comments on the manuscript.

Open Access. This article is distributed under the terms of the Creative Commons Attribution Noncommercial License which permits any noncommercial use, distribution, and reproduction in any medium, provided the original author(s) and source are credited.

\section{References}

[1] A. Arbey and F. Mahmoudi, SUSY constraints from relic density: high sensitivity to pre-BBN expansion rate, Phys. Lett. B 669 (2008) 46 [arXiv:0803.0741] [SPIRES].

[2] A. Arbey and F. Mahmoudi, SUSY constraints, relic density and very early universe, JHEP 05 (2010) 051 [arXiv: 0906.0368] [SPIRES].

[3] A. Arbey and F. Mahmoudi, LHC and ILC data and the early universe properties, Nuovo Cim. C 33 (2010) 151 [arXiv:1002.4096] [SPIRES].

[4] L. Randall and R. Sundrum, Out of this world supersymmetry breaking, Nucl. Phys. B 557 (1999) 79 [hep-th/9810155] [SPIRES].

[5] G.F. Giudice, M.A. Luty, H. Murayama and R. Rattazzi, Gaugino mass without singlets, JHEP 12 (1998) 027 [hep-ph/9810442] [SPIRES].

[6] A. Pomarol and R. Rattazzi, Sparticle masses from the superconformal anomaly, JHEP 05 (1999) 013 [hep-ph/9903448] [SPIRES].

[7] D.-W. Jung and J.Y. Lee, Anomaly-mediated supersymmetry breaking demystified, JHEP 03 (2009) 123 [arXiv: 0902.0464] [SPIRES].

[8] H. Baer et al., Gaugino anomaly mediated SUSY breaking: phenomenology and prospects for the LHC, JHEP 05 (2010) 069 [arXiv: 1002.4633] [SPIRES].

[9] K. Choi, K.S. Jeong and K.I. Okumura, Phenomenology of mixed modulus-anomaly mediation in fluxed string compactifications and brane models, JHEP 09 (2005) 039 [hep-ph/0504037] [SPIRES].

[10] R. Dermisek, H. Verlinde and L.-T. Wang, Hypercharged anomaly mediation, Phys. Rev. Lett. 100 (2008) 131804 [arXiv:0711.3211] [SPIRES]. 
[11] A. Basboll, M. Hindmarsh and D.R.T. Jones, Anomaly mediation and cosmology, arXiv:1101.5622 [SPIRES].

[12] H. Baer, R. Dermisek, S. Rajagopalan and H. Summy, Neutralino, axion and axino cold dark matter in minimal, hypercharged and gaugino AMSB, JCAP 07 (2010) 014 [arXiv: 1004.3297] [SPIRES].

[13] F.E. Paige, S.D. Protopopescu, H. Baer and X. Tata, ISAJET 7.69: a Monte Carlo event generator for $p p, \bar{p} p$ and $e^{+} e^{-}$reactions, hep-ph/0312045 [SPIRES].

[14] F. Mahmoudi, SuperIso: a program for calculating the isospin asymmetry of $B \rightarrow K^{*} \gamma$ in the MSSM, Comput. Phys. Commun. 178 (2008) 745 [arXiv:0710.2067] [SPIRES].

[15] F. Mahmoudi, SuperIso v2.3: a program for calculating flavor physics observables in supersymmetry, Comput. Phys. Commun. 180 (2009) 1579, online at http://superiso.in2p3.fr [arXiv: 0808.3144] [SPIRES].

[16] F. Mahmoudi, SuperIso v3.0, flavor physics observables calculations: Extension to NMSSM, Comput. Phys. Commun. 180 (2009) 1718 [SPIRES].

[17] A. Arbey and F. Mahmoudi, SuperIso Relic: a program for calculating relic density and flavor physics observables in supersymmetry, Comput. Phys. Commun. 181 (2010) 1277, oinline at http://superiso.in2p3.fr/relic [arXiv:0906.0369] [SPIRES].

[18] A. Arbey and F. Mahmoudi, SuperIso Relic v3.0: a program for calculating relic density and flavour physics observables: Extension to NMSSM, Comput. Phys. Commun. 182 (2011) 1582 [SPIRES].

[19] F. Mahmoudi, New constraints on supersymmetric models from $b \rightarrow s \gamma$, JHEP 12 (2007) 026 [arXiv: 0710.3791] [SPIRES].

[20] CDF collaboration, Search for $B_{s}^{0} \rightarrow \mu^{+} \mu^{-}$and $B_{d}^{0} \rightarrow \mu^{+} \mu^{-}$Decays in $3.7 f b^{-1}$ of $p \bar{p}$ Collisions with CDF II, CDF public note 9892 (2009).

[21] A.G. Akeroyd and F. Mahmoudi, Constraints on charged Higgs bosons from $D(s)^{ \pm} \rightarrow \mu^{ \pm} \nu$ and $D(s)^{ \pm} \rightarrow \tau^{ \pm} \nu$, JHEP 04 (2009) 121 [arXiv:0902.2393] [SPIRES].

[22] Flavianet Working Group on Kaon Decays collaboration, M. Antonelli et al., Precision tests of the standard model with leptonic and semileptonic kaon decays, arXiv:0801.1817 [SPIRES].

[23] WMAP collaboration, E. Komatsu et al., Seven-year Wilkinson Microwave Anisotropy Probe (WMAP) observations: cosmological interpretation, Astrophys. J. Suppl. 192 (2011) 18 [arXiv: 1001.4538] [SPIRES].

[24] H. Baer, R. Dermisek, S. Rajagopalan and H. Summy, Prospects for hypercharged anomaly mediated SUSY breaking at the LHC, JHEP 10 (2009) 078 [arXiv:0908.4259] [SPIRES].

[25] H. Baer, E.-K. Park, X. Tata and T.T. Wang, Collider and dark matter searches in models with mixed modulus-anomaly mediated SUSY breaking, JHEP 08 (2006) 041 [hep-ph/0604253] [SPIRES].

[26] M. Maniatis, The next-to-minimall supersymmetric extension of the standard model reviewed, Int. J. Mod. Phys. A 25 (2010) 3505 [arXiv:0906.0777] [SPIRES].

[27] U. Ellwanger, C. Hugonie and A.M. Teixeira, The next-to-minimal supersymmetric standard model, Phys. Rept. 496 (2010) 1 [arXiv:0910.1785] [SPIRES]. 
[28] U. Ellwanger and C. Hugonie, NMSPEC: a Fortran code for the sparticle and Higgs masses in the NMSSM with GUT scale boundary conditions, Comput. Phys. Commun. 177 (2007) 399 [hep-ph/0612134] [SPIRES].

[29] P. Gondolo and G. Gelmini, Cosmic abundances of stable particles: improved analysis, Nucl. Phys. B 360 (1991) 145 [SPIRES].

[30] J. Edsjo and P. Gondolo, Neutralino relic density including coannihilations, Phys. Rev. D 56 (1997) 1879 [hep-ph/9704361] [SPIRES].

[31] P. Salati, Quintessence and the relic density of neutralinos, Phys. Lett. B 571 (2003) 121 [astro-ph/0207396] [SPIRES].

[32] A. Arbey, in preparation, see http://superiso.in2p3.fr/relic/alterbbn.

[33] G.B. Gelmini and P. Gondolo, Neutralino with the right cold dark matter abundance in (almost) any supersymmetric model, Phys. Rev. D 74 (2006) 023510 [hep-ph/0602230] [SPIRES].

[34] G. Gelmini, P. Gondolo, A. Soldatenko and C.E. Yaguna, The effect of a late decaying scalar on the neutralino relic density, Phys. Rev. D 74 (2006) 083514 [hep-ph/0605016] [SPIRES].

[35] G.B. Gelmini and P. Gondolo, Neutralino with the right cold dark matter abundance in (almost) any supersymmetric model, Phys. Rev. D 74 (2006) 023510 [hep-ph/0602230] [SPIRES].

[36] G. Gelmini, P. Gondolo, A. Soldatenko and C.E. Yaguna, The effect of a late decaying scalar on the neutralino relic density, Phys. Rev. D 74 (2006) 083514 [hep-ph/0605016] [SPIRES].

[37] N. Okada and O. Seto, Relic density of dark matter in brane world cosmology, Phys. Rev. D 70 (2004) 083531 [hep-ph/0407092] [SPIRES].

[38] K. Jedamzik, Big Bang nucleosynthesis constraints on hadronically and electromagnetically decaying relic neutral particles, Phys. Rev. D 74 (2006) 103509 [hep-ph/0604251] [SPIRES].

[39] W. Beenakker et al., The Production of charginos/neutralinos and sleptons at hadron colliders, Phys. Rev. Lett. 83 (1999) 3780 [Erratum ibid. 100 (2008) 029901] [hep-ph/9906298] [SPIRES].

[40] H. Baer and X. Tata, Probing charginos and neutralinos beyond the reach of LEP at the Tevatron collider, Phys. Rev. D 47 (1993) 2739 [SPIRES].

[41] H. Baer, C.-h. Chen, F. Paige and X. Tata, Trileptons from chargino-neutralino production at the CERN Large Hadron Collider, Phys. Rev. D 50 (1994) 4508 [hep-ph/9404212] [SPIRES]. 